\title{
Helly-gap of a graph and vertex eccentricities
}

\author{
Feodor F. Dragan and Heather M. Guarnera \\ Algorithmic Research Laboratory, Department of Computer Science \\ Kent State University, Kent, Ohio, USA \\ dragan@cs.kent.edu, hmichaud@kent.edu
}

May 6, 2020

\begin{abstract}
A new metric parameter for a graph, Helly-gap, is introduced. A graph $G$ is called $\alpha$-weaklyHelly if any system of pairwise intersecting disks in $G$ has a nonempty common intersection when the radius of each disk is increased by an additive value $\alpha$. The minimum $\alpha$ for which a graph $G$ is $\alpha$-weakly-Helly is called the Helly-gap of $G$ and denoted by $\alpha(G)$. The Helly-gap of a graph $G$ is characterized by distances in the injective hull $\mathcal{H}(G)$, which is a (unique) minimal Helly graph which contains $G$ as an isometric subgraph. This characterization is used as a tool to generalize many eccentricity related results known for Helly graphs $(\alpha(G)=0)$, as well as for chordal graphs $(\alpha(G) \leq 1)$, distance-hereditary graphs $(\alpha(G) \leq 1)$ and $\delta$-hyperbolic graphs $(\alpha(G) \leq 2 \delta)$, to all graphs, parameterized by their Helly-gap $\alpha(G)$. Several additional graph classes are shown to have a bounded Helly-gap, including AT-free graphs and graphs with bounded tree-length, bounded chordality or bounded $\alpha_{i}$-metric.
\end{abstract}

Keywords: Helly-type property; injective hull; chordal graphs; $\delta$-hyperbolic graphs; tree-length; $\alpha_{i}$-metric, eccentricity; center; diameter; radius.

\section{Introduction}

All graphs in this paper are unweighted, undirected, connected, and without loops or multiple edges. In a graph $G=(V(G), E(G))$, a disk $D_{G}(v, r)$ with radius $r$ and centered at a vertex $v$ consists of all vertices with distance at most $r$ from $v$, i.e., $D_{G}(v, r)=\left\{u \in V(G): d_{G}(u, v) \leq r\right\}$. A graph $G$ is called Helly if every system of pairwise intersecting disks of $G$ has a non-empty intersection.

Definition 1 (Helly graph). A graph $G$ is Helly if, for any system of disks $\mathcal{F}=\left\{D_{G}(v, r(v)): v \in S \subseteq\right.$ $V(G)\}$, the following Helly property holds: if $X \cap Y \neq \emptyset$ for every $X, Y \in \mathcal{F}$, then $\bigcap_{v \in S} D_{G}(v, r(v)) \neq \emptyset$.

Helly graphs are well investigated. They have several characterizations and important features as established in [4, 5, 16, 17, 44, 46]. They are exactly the so-called absolute retracts of reflexive graphs and possess a certain elimination scheme [4, 5, 16, 17, 44]. They can be recognized in $O\left(n^{2} m\right)$ time [16] where $n$ is the number of vertices and $m$ is the number of edges. The Helly property works as a compactness criterion on graphs [46]. More importantly, every graph is isometrically embeddable into a Helly graph [27,36, 40] (see Section 3 for more details).

Many nice properties of Helly graphs are based on the eccentricity $e_{G}(v)$ of a vertex $v$, which is defined as the maximum distance from $v$ to any other vertex of the graph (i.e., $e_{G}(v)=$ $\max _{u \in V(G)} d_{G}(v, u)$ ). The minimum (maximum) eccentricity in a graph $G$ is the radius $\operatorname{rad}(G)$ (diameter $\operatorname{diam}(G))$. The center $C(G)$ of a graph $G$ is the set of all vertices with minimum eccentricity (i.e., $C(G)=\left\{v \in V(G): e_{G}(v)=\operatorname{rad}(G)\right\}$. Graph's diameter is tightly bounded in Helly graphs as $2 \operatorname{rad}(G) \geq \operatorname{diam}(G) \geq 2 \operatorname{rad}(G)-1$ [16,17]. Moreover, the eccentricity function in Helly graphs is 
unimodal [17], that is, any local minimum coincides with the global minimum; this is equivalent to the condition that, for any vertex $v \in V(G), e_{G}(v)=d_{G}(v, C(G))+\operatorname{rad}(G)$ holds [16]. The unimodality of the eccentricity function was recently used in [28,29] to compute the radius, diameter and a central vertex of a Helly graph in subquadratic time. For a vertex $v \in V(G)$, let $F_{G}(v)$ denote the set of all vertices farthest from $v$, that is, $F_{G}(v)=\left\{u \in V(G): e_{G}(v)=d_{G}(v, u)\right\}$. For every vertex $v$ of a Helly graph $G$, each vertex $u \in F_{G}(v)$ satisfies $e_{G}(u) \geq 2 \operatorname{rad}(G)-\operatorname{diam}(C(G))$ [16]. Although the center $C(G)$ of a Helly graph $G$ may have an arbitrarily large diameter (as any Helly graph is the center of some Helly graph), $C(G)$ induces a Helly graph and is isometric in $G$ [16]. Additionally, any power of a Helly graph is a Helly graph as well [16.

In this paper, we introduce a far reaching generalization of Helly graphs, $\alpha$-weakly-Helly graphs. We define $\alpha$-weakly-Helly graphs as those graphs which are "weakly" Helly with the following simple generalization: for any system of disks, if the disks pairwise intersect, then by expanding the radius of each disk by some integer $\alpha$ there forms a common intersection. Thus, Helly graphs are exactly 0-weakly-Helly graphs.

Definition 2 ( $\alpha$-weakly-Helly graph). A graph $G$ is $\alpha$-weakly-Helly if, for any system of disks $\mathcal{F}=$ $\left\{D_{G}(v, r(v)): v \in S \subseteq V(G)\right\}$, the following $\alpha$-weakly-Helly property holds: if $X \cap Y \neq \emptyset$ for every $X, Y \in \mathcal{F}$, then $\bigcap_{v \in S} D_{G}(v, r(v)+\alpha) \neq \emptyset$.

Clearly, every graph is $\alpha$-weakly-Helly for some $\alpha$. We call the minimum $\alpha$ for which a graph $G$ is $\alpha$-weakly-Helly the Helly-gap of $G$, denoted by $\alpha(G)$.

Interestingly, there are a few results in the literature which demonstrate that such a $\alpha$-weakly-Helly property with bounded $\alpha$ is present in some graphs and in some metric spaces. In [14, Chepoi and Estellon showed that for every $\delta$-hyperbolic geodesic space $(X, d)$ (and for every $\delta$-hyperbolic graph $G$ ), if disks of the family $\mathcal{F}=\{D(x, r(x)): v \in S \subseteq X, S$ is compact $\}$ pairwise intersect, then the disks $\{D(x, r(x)+2 \delta): x \in S\}$ have a nonempty common intersection (see also [11]). That is, the disks in $\delta$-hyperbolic geodesic spaces and in $\delta$-hyperbolic graphs satisfy $(2 \delta)$-weakly-Helly property. Even earlier, Lenhart, Pollack et al. [41, Lemma 9] established that the disks of simple polygons endowed with the link distance satisfy a Helly-type property which implies 1-weakly-Helly property. For chordal graphs [20] and for distance-hereditary graphs [18] (as well as for a more general class of graphs [10]) the following similar result is known: if for a set $M \subseteq V(G)$ and radius function $r: M \rightarrow \mathbb{N}$, every two vertices $x, y \in M$ satisfy $d_{G}(x, y) \leq r(x)+r(y)+1$, then there is a clique $K$ in $G$ such that $d_{G}(v, K) \leq r(v)$ for all $v \in M$. Hence, clearly, every chordal graph and every distance-hereditary graph is 1-weakly-Helly. Note that two disks $D_{G}(v, p)$ and $D_{G}(u, q)$ intersect if and only if $d_{G}(v, u) \leq p+q$.

There are numerous other approaches to generalize in some form the Helly graphs. One may loosen the restriction on the type of sets which must satisfy the Helly property. If one considers neighborhoods or cliques, rather than arbitrary disks, then one gets the neighborhood-Helly graphs and the clique-Helly graphs as superclasses of Helly graphs (see [6, 34, 42] and papers cited therein). One may also generalize the Helly-property that a family of sets satisfies. This can be accomplished by specifying a minimum number or size of sets that pairwise intersect, a minimum number of subfamilies that have a common intersection, or the size of the intersection (see [30] and papers cited therein). Far reaching examples include the $(p, q)$-Helly property (see [2,3] and papers cited therein) and the fractional Helly property (see [7,37] and papers cited therein). There is also a related to ours notion of $\lambda$-hyperconvexity in metric spaces $(X, d)$ 31, 38, where $\lambda$ is the smallest multiplicative constant such that for any system of disks $\mathcal{F}=\{D(x, r(x)): x \in S \subseteq X\}$, the following property holds: if $X \cap Y \neq \emptyset$ for every $X, Y \in \mathcal{F}$, then $\bigcap_{x \in S} D(x, \lambda \cdot r(x)) \neq \emptyset$. Several classical metric spaces are $\lambda$-hyperconvex for a bounded $\lambda$ (see [31] and papers cited therein). These include reflexive Banach spaces and dual Banach spaces $(\lambda \leq 2)$ and Hilbert spaces $(\lambda \leq \sqrt{2})$. The classical Jung Theorem asserting that each 
subset $S$ of the Euclidean space $\mathbb{E}^{m}$ with finite diameter $D$ is contained in a disk of radius at most $\sqrt{\frac{m}{2(m+1)}} D$ belongs to this kind of results, too.

In this paper, we are interested in $\alpha$-weakly-Helly graphs, where $\alpha$ describes an additive constant by which radius of each disk in the family of pairwise intersecting disks can be increased in order to obtain a nonempty common intersection of all expanded disks. By definition, any $\alpha$-weakly-Helly graph is $(\alpha+1)$-hyperconvex. We find that there are also an abundance of graph classes with a bounded Hellygap $\alpha(G)$. Here, we generalize many eccentricity related results known for Helly graphs (as well as for chordal graphs, distance-hereditary graphs and $\delta$-hyperbolic graphs) to all graphs, parameterized by their Helly-gap $\alpha(G)$. We provide a characterization of weakly-Helly graphs with respect to their injective hulls and use this as a tool to prove those generalizations. Several additional well-known graph classes that are $\alpha$-weakly-Helly for some constant $\alpha$ are also identified.

The main results of this paper can be summarized as follows. After providing in Section 2 necessary definitions, notations and some relevant results on Helly graphs, in Section 3 we give a characterization of $\alpha$-weakly-Helly graphs through distances in injective hulls. The injective hull of a graph $G$, denoted by $\mathcal{H}(G)$, is a (unique) minimal Helly graph which contains $G$ as an isometric subgraph [27, 36]. We show that $G$ is an $\alpha$-weakly-Helly graph if and only if for every vertex $h \in V(\mathcal{H}(G))$ there is a vertex $v \in V(G)$ such that $d_{\mathcal{H}(G)}(h, v) \leq \alpha$ (Theorem 11). In Section 4, we relate the diameter, radius, and all eccentricities in $G$ to their counterparts in $H:=\mathcal{H}(G)$. In particular, we show that $e_{G}(v)=e_{H}(v)$ for all $v \in V(G), \operatorname{diam}(G)=\operatorname{diam}(H)$, and $\operatorname{rad}(G)-\alpha(G) \leq \operatorname{rad}(H) \leq \operatorname{rad}(G)$. Additionally, we show $\operatorname{diam}_{G}(C(G))-2 \alpha(G) \leq \operatorname{diam}_{H}(C(H)) \leq \operatorname{diam}_{G}\left(C^{\alpha(G)}(G)\right)+2 \alpha(G)$, where $C^{\ell}(G)=\{v \in V(G)$ : $\left.e_{G}(v) \leq \operatorname{rad}(G)+\ell\right\}$. We also provide several bounds on $\alpha(G)$ including its relation to diameter and radius as well as investigate the Helly-gap of powers of weakly-Helly graphs (Theorem 3). In particular, $\lfloor(2 \operatorname{rad}(G)-\operatorname{diam}(G)) / 2\rfloor \leq \alpha(G) \leq\lfloor\operatorname{diam}(G) / 2\rfloor$ holds. The eccentricity function in $\alpha$ weakly-Helly graphs is shown to exhibit the property that any vertex $v \notin C^{\alpha}(G)$ has a nearby vertex, within distance $2 \alpha+1$ from $v$, with strictly smaller eccentricity (Theorem 44). In Section 5, we give upper and lower bounds on the eccentricity $e_{G}(v)$ of a vertex $v$. We consider bounds based on the distance from $v$ to a closest vertex in $C^{\alpha(G)}(G)$, whether $v$ is farthest from some other vertex and if $\operatorname{diam}_{G}\left(C^{\alpha(G)}(G)\right)$ is bounded. In particular, we show that $\left|e_{G}(v)-d_{G}\left(v, C^{\alpha(G)}(G)\right)-\operatorname{rad}(G)\right| \leq \alpha(G)$ holds for any vertex $v \in V(G)$ (Theorem 5 ). We also prove the existence of a spanning tree $T$ of $G$ which gives an approximation of all vertex eccentricities in $G$ with an additive error depending only on $\alpha(G)$ and $\operatorname{diam}_{G}\left(C^{\alpha(G)}(G)\right.$ ) (Theorem 7). We find that in any shortest path to $C^{\alpha(G)}(G)$, the number of vertices with locality more than 1 does not exceed $2 \alpha(G)$, where the locality of a vertex $v$ is the minimum distance from $v$ to a vertex of strictly smaller eccentricity (Theorem 8 ). All these results greatly generalize some known facts about distance-hereditary graphs, chordal graphs, and $\delta$-hyperbolic graphs. Those graphs have bounded Helly gap. In Section 6, we identify several more (well-known) graph classes with a bounded Helly-gap, including $k$-chordal graphs, AT-free graphs, rectilinear grids, graphs with a bounded $\alpha_{i}$-metric, and graphs with bounded tree-length or treebreadth. We conclude with open questions in Section 7 ,

\section{Preliminaries}

All graphs $G=(V(G), E(G))$ occurring in this paper are undirected, connected, and without loops or multiple edges. The length of a path from a vertex $u$ to a vertex $v$ is the number of edges in the path. The distance $d_{G}(u, v)$ between two vertices $u$ and $v$ is the length of a shortest path connecting them in $G$. The distance from a vertex $u$ to a vertex set $S \subseteq V(G)$ is defined as $d_{G}(u, S)=\min _{s \in S} d_{G}(u, s)$. The eccentricity of a vertex is defined as $e_{G}(v)=\max _{u \in V(G)} d_{G}(v, u)$. The vertex set $F_{G}(v)$ for a vertex $v \in V(G)$ denotes the set of all vertices farthest from $v$, that is, $F_{G}(v)=\left\{u \in V(G): e_{G}(v)=\right.$ $\left.d_{G}(v, u)\right\}$. A disk of radius $k$ centered at a set $S$ (or a vertex) is the set of vertices of distance at most $k$ from $S$, that is, $D_{G}(S, k)=\left\{u \in V(G): d_{G}(u, S) \leq k\right\}$. We omit $G$ from subindices when the 
graph is known by context.

The $k^{\text {th }}$ power $G^{k}$ of a graph $G$ is a graph that has the same set of vertices, but in which two distinct vertices are adjacent if and only if their distance in $G$ is at most $k$. A subgraph $G^{\prime}$ of a graph $G$ is called isometric (or a distance preserving subgraph) if for any two vertices $x, y$ of $G^{\prime}$, $d_{G}(x, y)=d_{G^{\prime}}(x, y)$ holds. The interval $I(x, y)$ is the set of all vertices belonging to a shortest $(x, y)$ path, that is, $I(x, y)=\{u \in V(G): d(x, u)+d(u, y)=d(x, y)\}$. The interval slice $S_{k}(x, y)$ is the set of vertices on $I(x, y)$ which are at distance $k$ from vertex $x$. An interval $I(x, y)$ is said to be $\kappa$-thin if, for any $0 \leq \ell \leq d(x, y)$ and any $u, v \in S_{\ell}(x, y), d(u, v) \leq \kappa$ holds. The smallest $\kappa$ for which all intervals of $G$ are $\kappa$-thin is called the interval thinness of $G$ and denoted by $\kappa(G)$. By $G-\{x\}$ we denote an induced subgraph of $G$ obtained from $G$ by removing a vertex $x \in V(G)$.

The minimum (maximum) eccentricity in a graph $G$ is the $\operatorname{radius} \operatorname{rad}(G)$ (diameter diam $(G)$ ). The center $C(G)$ is the set of vertices with minimum eccentricity (i.e., $C(G)=\{v \in V(G): e(v)=$ $\operatorname{rad}(G)\}$. It will be useful to define also $C^{k}(G)=\{v \in V(G): e(v) \leq \operatorname{rad}(G)+k\}$; then $C(G)=$ $C^{0}(G)$. Let $M \subseteq V(G)$ be a subset of vertices of $G$. We distinguish the eccentricity with respect to $M$ as follows: denote by $e_{G}^{M}(v)$ the maximum distance from vertex $v$ to any vertex $u \in M$, i.e., $e_{G}^{M}(v)=\max _{u \in M} d_{G}(v, u)$. We then define $F_{G}^{M}(v)=\left\{u \in M: e_{G}^{M}(v)=d_{G}(v, u)\right\}, \operatorname{rad}_{G}(M)=$ $\min _{v \in V(G)} e_{G}^{M}(v)$, and $\operatorname{diam}_{G}(M)=\max _{v \in M} e_{G}^{M}(v)$. Let $C_{G}^{\ell}(M)=\left\{v \in V(G): e_{G}^{M}(v) \leq \operatorname{rad}_{G}(M)+\right.$ $\ell\}$ and $C_{G}(M)=C_{G}^{0}(M)$. For simplicity, when $M=V(G)$, we continue to use the notation $\operatorname{rad}(G)$, $C(G)$, and $\operatorname{diam}(G)$.

The following results for Helly graphs will prove useful for $\alpha$-weakly-Helly graphs.

Lemma 1. [16] Let $G$ be a Helly graph. For every $M \subseteq V(G)$, the graph induced by the center $C_{G}(M)$ is Helly and is an isometric subgraph of $G$.

Given this lemma, it will be convenient to also denote by $C_{G}(M)$ a subgraph of $G$ induced by $C_{G}(M)$.

Lemma 2. [4, 16] Let $G$ be a Helly graph. If there are two distinct vertices $w, x \in V(G)$ such that $D(w, 1) \supseteq D(x, 1)$, then $G-\{x\}$ is Helly and an isometric subgraph of $G$.

For a graph $G$ and a subset $M \subseteq V(G)$, the eccentricity function $e_{G}^{M}(\cdot)$ is called unimodal if every vertex $v \in V(G) \backslash C_{G}(M)$ has a neighbor $u$ such that $e_{G}^{M}(u)<e_{G}^{M}(v)$.

Lemma 3. [16, 17] A graph $G$ is Helly if and only if the eccentricity function $e_{G}^{M}(\cdot)$ is unimodal on $G$ for every $M \subseteq V(G)$.

The following two results were earlier proven in [16] only for $M=V(G)$ and then later extended in 21] to all $M \subseteq V(G)$.

Lemma 4. [21] Let $G$ be a Helly graph. For any $M \subseteq V(G), \operatorname{rrad}_{G}(M)-1 \leq \operatorname{diam}_{G}(M) \leq$ $\operatorname{2rad}_{G}(M)$. Moreover, $\operatorname{rad}_{G}(M)=\left\lceil\operatorname{diam}_{G}(M) / 2\right\rceil$.

Lemma 5. [21] Let $G$ be a Helly graph. For every $v \in V(G)$ and $M \subseteq V(G)$, $e_{G}^{M}(v)=$ $d_{G}\left(v, C_{G}(M)\right)+\operatorname{rad}_{G}(M)$ holds.

Corollary 1. For every Helly graph $G$, any subset $M \subseteq V(G)$ and any integers $\ell \geq 0$ and $k \geq 0, C_{G}^{\ell+k}(M)=D_{G}\left(C_{G}^{k}(M)+\ell\right)=D_{G}\left(C_{G}(M)+k+\ell\right)$. Furthermore, diam ${ }_{G}\left(C_{G}^{\ell+\bar{k}}(M)\right) \leq$ $\operatorname{diam}_{G}\left(C_{G}^{k}(M)\right)+2 \ell$.

Proof. Consider a vertex $v$ with $e_{G}^{M}(v)=k+\ell+\operatorname{rad}_{G}(M)$ and a vertex $c$ in $C_{G}(M)$ closest to $v$. By Lemma 5, $e_{G}^{M}(v)=d_{G}\left(v, C_{G}(M)\right)+\operatorname{rad}_{G}(M)=d_{G}(v, c)+\operatorname{rad}_{G}(M)=k+\ell+\operatorname{rad}_{G}(M)$. Hence, for every vertex $v, d_{G}\left(v, C_{G}(M)\right)=k+\ell$ if and only if $e_{G}^{M}(v)=k+\ell+\operatorname{rad}_{G}(M)$.

Let $u$ be a vertex on a shortest path from $v$ to $c$ at distance $k$ from $c$. By Lemma 5, $e_{G}^{M}(u)=$ $d_{G}(u, c)+\operatorname{rad}_{G}(M)=k+\operatorname{rad}_{G}(M)$ and hence $e_{G}^{M}(v)=k+\ell+\operatorname{rad}_{G}(M)=e_{G}^{M}(u)+\ell$. Therefore, $e_{G}^{M}(v)=k+\ell+\operatorname{rad}_{G}(M)$ if and only if $d_{G}\left(v, C_{G}^{k}(M)\right)=d_{G}(v, u)=\ell$. 
Let $x, y$ be vertices of $C_{G}^{\ell+k}(M)$ with $d_{G}(x, y)=\operatorname{diam}_{G}\left(C_{G}^{\ell+k}(M)\right)$. Since $d_{G}\left(v, C_{G}^{k}(M)\right) \leq \ell$ for each $v \in\{x, y\}$, by the triangle inequality, we have $d_{G}(x, y) \leq d_{G}\left(x, C_{G}^{k}(M)\right)+\operatorname{diam}_{G}\left(C_{G}^{k}(M)\right)+$ $d_{G}\left(y, C_{G}^{k}(M)\right) \leq \operatorname{diam}_{G}\left(C_{G}^{k}(M)\right)+2 \ell$.

\section{Distances in injective hulls characterize weakly-Helly graphs}

Recall that the injective hull of $G$, denoted by $\mathcal{H}(G)$, is a minimal Helly graph which contains $G$ as an isometric subgraph [27, 36]. It turns out that $\mathcal{H}(G)$ is unique for every $G$ [36]. When $G$ is known by context, we often let $H:=\mathcal{H}(G)$. By an equivalent definition of an injective hull [27] (also called a tight span), each vertex $f \in V(\mathcal{H}(G))$ can be represented as a vector with nonnegative integer values $f(x)$ for each $x \in V(G)$, such that the following two properties hold:

$$
\begin{gathered}
\forall x, y \in V(G) f(x)+f(y) \geq d_{G}(x, y) \\
\forall x \in V(G) \exists y \in V(G) f(x)+f(y)=d_{G}(x, y)
\end{gathered}
$$

Additionally, there is an edge between two vertices $f, g \in V(\mathcal{H}(G))$ if and only if their Chebyshev distance is 1, i.e., $\max _{x \in V(G)}|f(x)-g(x)|=1$. Thus, $d_{H}(f, g)=\max _{x \in V(G)}|f(x)-g(x)|$. Notice that if $f \in V(\mathcal{H}(G))$, then $\{D(x, f(x)): x \in V(G)\}$ is a family of pairwise intersecting disks. For a vertex $z \in V(G)$, define the distance function $d_{z}$ by setting $d_{z}(x)=d_{G}(z, x)$ for any $x \in V(G)$. By the triangle inequality, each $d_{z}$ belongs to $V(\mathcal{H}(G))$. An isometric embedding of $G$ into $\mathcal{H}(G)$ is obtained by mapping each vertex $z$ of $G$ to its distance vector $d_{z}$.

We classify every vertex $v$ in $V(\mathcal{H}(G))$ as either a real vertex or a Helly vertex. A vertex $f \in$ $V(\mathcal{H}(G))$ is a real vertex provided $f=d_{z}$ for some $z \in V(G)$, i.e., there is a one-to-one correspondence between $z \in V(G)$ and its representative real vertex $f \in V(\mathcal{H}(G))$ which uniquely satisfies $f(z)=0$ and $f(x)=d_{G}(z, x)$ for all $x \in V(G)$. When working with $\mathcal{H}(G)$, we will use interchangeably the notation $V(G)$ to represent the vertex set in $G$ as well as the vertex subset of $\mathcal{H}(G)$ which uniquely corresponds to the vertex set of $G$. Then, a vertex $v \in V(\mathcal{H}(G))$ is a real vertex if it belongs to $V(G)$ and a Helly vertex otherwise. Equivalently, a vertex $h \in V(\mathcal{H}(G))$ is a Helly vertex provided that $h(x) \geq 1$ for all $x \in V(G)$, that is, a Helly vertex exists only in the injective hull $\mathcal{H}(G)$ and not in $G$. We will show in Theorem 1 that a graph $G$ is $\alpha$-weakly-Helly if and only if the distance from any Helly vertex in $\mathcal{H}(G)$ to a closest real vertex in $V(G)$ is no more than $\alpha$. We recently learned that this result was independently discovered by Chalopin et al. [8]. They use name coarse Helly property for $\alpha$-weakly-Helly property used here.

The following properties will be useful to the main result of this section and to later sections. A vertex $x$ is called a peripheral vertex if $I(y, x) \not \subset I(y, z)$ for some vertex $y$ and all vertices $z \neq x$. We show next that the peripheral vertices of $\mathcal{H}(G)$ are real vertices. This adheres to the intuitive notion that an injective hull contains all of the Helly vertices "between" the vertices of $G$, so that the outermost vertices of $\mathcal{H}(G)$ are real.

Proposition 1. Peripheral vertices of $\mathcal{H}(G)$ are real.

Proof. By contradiction, suppose there is a peripheral Helly vertex $u \in V(\mathcal{H}(G)) \backslash V(G)$. By definition, there is a vertex $s \in V(\mathcal{H}(G))$ such that, for all $x \in V(\mathcal{H}(G)), I(s, u) \not \subset I(s, x)$. Let $k:=d(u, s)$. Consider pairwise intersecting disks $D(s, k-1)$ and $D(x, 1)$ for each $x \in D(u, 1)$. By the Helly property, there exists vertex $w \in V(\mathcal{H}(G))$ with $d(w, s)=k-1$ and $D(w, 1) \supseteq D(u, 1)$. By Lemma 2 , $\mathcal{H}(G)-\{u\}$ is Helly and is an isometric subgraph of $\mathcal{H}(G)$. Since $u$ is a Helly vertex, $G$ is an isometric subgraph of $\mathcal{H}(G)-\{u\}$, a contradiction with the minimality of $\mathcal{H}(G)$.

Moreover, we show that any shortest path of $\mathcal{H}(G)$ is a subpath of a shortest path between real vertices, which will later prove a useful property of injective hulls. 
Proposition 2. Let $H$ be the injective hull of $G$. For any shortest path $P(x, y)$, where $x, y \in V(H)$, there is a shortest path $P\left(x^{\prime}, y^{\prime}\right)$, where $x^{\prime}, y^{\prime} \in V(G)$ such that $P\left(x^{\prime}, y^{\prime}\right) \supseteq P(x, y)$.

Proof. If $x$ and $y$ are both real vertices, then the proposition is trivially true. Without loss of generality, let $y$ be a Helly vertex. Consider a breadth-first search layering where $y$ belongs to layer $L_{i}$ of $\operatorname{BFS}(H, x)$. Let $y^{\prime} \in L_{k}$ be a vertex with $y \in I\left(x, y^{\prime}\right)$ that maximizes $k=d_{H}\left(x, y^{\prime}\right)$. Then, for any vertex $z \in V(\mathcal{H}(G)), I\left(x, y^{\prime}\right) \not \subset I(x, z)$. By Proposition 1, $y^{\prime} \in V(G)$. If $x \notin V(G)$, then applying the previous step using $\operatorname{BFS}\left(H, y^{\prime}\right)$ yields vertex $x^{\prime} \in V(G)$.

We are now ready to prove the main result of this section.

Theorem 1. For any vertex $h \in V(\mathcal{H}(G))$ there is a real vertex $v \in V(G)$ such that $d_{\mathcal{H}(G)}(h, v) \leq \alpha$ if and only if $G$ is an $\alpha$-weakly-Helly graph.

Proof. Suppose any Helly vertex in $H:=\mathcal{H}(G)$ is within distance at most $\alpha$ from a vertex of $G$. Consider in $G$ a family of pairwise intersecting disks $\mathcal{F}_{G}=\left\{D_{G}(v, r(v)): v \in S \subseteq V(G)\right\}$. As $H$ contains $G$ as an isometric subgraph, the disks $\mathcal{F}_{H}=\left\{D_{H}(v, r(v): v \in S\}\right.$ are also pairwise intersecting in $H$. By the Helly property, in $H$ there is a vertex $x \in \bigcap_{v \in S} D_{H}(v, r(v))$. By assumption, there is a vertex $u \in V(G)$ such that $d_{H}(u, x) \leq \alpha$. Thus $u \in \bigcap_{v \in S} D_{G}(v, r(v)+\alpha)$ and so $G$ is $\alpha$-weakly-Helly.

Assume now that $G$ is $\alpha$-weakly-Helly. Let $h \in V(H)$ be an arbitrary vertex represented as a vector with nonnegative integer values $h(x)$ for each $x \in V(G)$ satisfying conditions (11) and (2) from the definition of an injective hull. Then, $\left\{D_{G}(x, h(x)): x \in V(G)\right\}$ is a family of pairwise intersecting disks in $G$. By the $\alpha$-weakly-Helly property, there is a real vertex $z \in V(G)$ belonging to $\bigcap_{x \in V(G)} D_{G}(x, h(x)+\alpha)$. To establish that $d_{H}(z, h) \leq \alpha$ and complete the proof, we will show that $\max _{t \in V(G)}|z(t)-h(t)| \leq \alpha$.

First, we show that $z(t)-h(t) \leq \alpha$ for all $t \in V(G)$. As $z$ is a real vertex, by definition, $z(t)=$ $d_{G}(z, t)$ and, by the $\alpha$-weakly-Helly property (recall that $z \in \bigcap_{x \in V(G)} D_{G}(x, h(x)+\alpha)$ ), $z(t) \leq h(t)+\alpha$. Next, we show that $h(t)-z(t) \leq \alpha$ for all $t \in V(G)$. Suppose that $h(x)-z(x)>\alpha$ for some $x \in V(G)$. Then, for all vertices $y \neq x, h(x)+h(y)>z(x)+\alpha+h(y) \geq z(x)+z(y) \geq d(x, y)$, a contradiction with condition (2).

The injective hull is also useful to prove that $\alpha$-weakly-Helly graphs are closed under pendant vertex addition.

Lemma 6. Let $G+\{x\}$ be the graph obtained from $G$ by adding a vertex $x$ pendant to any fixed vertex $v \in V(G)$. Then, $\mathcal{H}(G+\{x\})=\mathcal{H}(G)+\{x\}$.

Proof. As $x$ is pendant to $v$, then all $u \in V(G)$ have $d_{G+\{x\}}(u, x)=d_{G}(u, v)+1$. Let $H_{1}:=\mathcal{H}(G+\{x\})$ and $H_{2}:=\mathcal{H}(G)+\{x\}$. Note that $H_{2}$ is a Helly graph containing $G+\{x\}$ as an isometric subgraph. We first show that any $h \in V\left(H_{1}\right)$ also belongs to $V\left(H_{2}\right)$. The statement clearly holds if $h$ is a real vertex, so assume that $h$ is a Helly vertex of $H_{1}$ represented as a vector with nonnegative integer values for each $u \in V(G+\{x\})$ satisfying conditions (11) and (2) from the definition of an injective hull. We will show $h$ also satisfies the conditions under $G$. By condition (1) under $G+\{x\}$, and since $G$ is isometric in $G+\{x\}$, for all $u, y \in V(G), h(u)+h(y) \geq d_{G+\{x\}}(u, y)=d_{G}(u, y)$. Thus, $h$ satisfies condition (11) in $G$. By condition (2) under $G+\{x\}$, for every $u \in V(G)$ there is a vertex $y \in V(G+\{x\})$ with $h(u)+h(y)=d_{G+\{x\}}(u, y)$. We claim that if $y=x$, then $h(x)=h(v)+1$ and so vertex $v$ also satisfies $h(u)+h(v)=h(u)+h(y)-1=d_{G+\{x\}}(u, y)-1=d_{G}(u, v)$. On one hand, $h(x) \leq h(v)+1$ since $h(u)+h(x)=d_{G+\{x\}}(u, x)=d_{G}(u, v)+1 \leq h(u)+h(v)+1$. On the other hand, let $z \in V(G) \cup\{x\}$ be a vertex such that $h(z)+h(v)=d_{G+\{x\}}(z, v)$. Note that $z \neq x$ as $h$ is not a real vertex and therefore $h(v)=d_{H_{1}}(h, v)>0$ and $h(z)=d_{H_{1}}(h, z)>0$. Then, $h(x) \geq d_{G+\{x\}}(z, x)-h(z)=d_{G+\{x\}}(z, v)+1-h(z)=h(v)+1$. With the claim established, $h$ satisfies condition (2) in $G$. Thus, $h \in V\left(H_{2}\right)$ and $V\left(H_{1}\right) \subseteq V\left(H_{2}\right)$. By minimality of $\mathcal{H}(G)$, $V\left(H_{1}\right)=V\left(H_{2}\right)$. 
Corollary 2. Let $G+\{x\}$ be the graph obtained from $G$ by adding a pendant vertex $x$ adjacent to any fixed vertex $v \in V(G)$. Then, $\alpha(G)=\alpha(G+\{x\})$.

\section{Diameter, radius, and all eccentricities}

We establish several bounds on all eccentricities, and the diameter and radius in particular, for $\alpha$ weakly-Helly graphs. As an intermediate step, we relate the diameter, radius, and all eccentricities in $G$ to their counterparts in $\mathcal{H}(G)$.

\subsection{Eccentricities and centers}

First, we provide a few immediate consequences of Proposition 1 which establishes that farthest vertices in $\mathcal{H}(G)$ are real vertices. That is, for any $v \in V(\mathcal{H}(G)), F_{\mathcal{H}(G)}(v) \subseteq V(G)$. It follows that all eccentricities in $G$ and the diameter of $G$ are preserved in $\mathcal{H}(G)$, including with respect to any subset $M \subseteq V(G)$ of real vertices.

Proposition 3. Let $H$ be the injective hull of $G$. For any $M \subseteq V(G)$ and $v \in V(G), e_{G}^{M}(v)=e_{H}^{M}(v)$. Moreover, $e_{G}(v)=e_{H}(v)$.

Proposition 4. Let $H$ be the injective hull of $G$. For any $M \subseteq V(G)$, $\operatorname{diam}_{G}(M)=\operatorname{diam}_{H}(M)$. Moreover, $\operatorname{diam}(G)=\operatorname{diam}(H)$.

Proposition 5. Let $H$ be the injective hull of an $\alpha$-weakly-Helly graph $G$. For any $M \subseteq V(G)$, $\operatorname{rad}_{G}(M)-\alpha \leq \operatorname{rad}_{H}(M) \leq \operatorname{rad}_{G}(M)$. In particular, $\operatorname{rad}(G)-\alpha \leq \operatorname{rad}(H) \leq \operatorname{rad}(G)$.

Proof. By Proposition 3, the eccentricity of any vertex of $G$ is preserved in $H$. Hence, $\operatorname{rad}_{H}(M) \leq$ $\operatorname{rad}_{G}(M)$. Consider any vertex $h \in C_{H}(M)$. We have $d_{H}(h, x) \leq \operatorname{rad}_{H}(M)$ for all $x \in M$. By Theorem 1, there is a real vertex $v \in V(G)$ such that $d_{H}(v, h) \leq \alpha$. By the triangle inequality, $\operatorname{rad}_{G}(M) \leq e_{G}^{M}(v) \leq \max _{x \in M}\left\{d_{H}(v, h)+d_{H}(h, x)\right\} \leq \operatorname{rad}_{H}(M)+\alpha$.

Though the diameter is preserved, the radius in $\mathcal{H}(G)$ may be smaller than the radius in $G$ by at most $\alpha(G)$. In this case, the radius in $\mathcal{H}(G)$ is realized by Helly vertices which are not present in $G$, resulting in different centers in $\mathcal{H}(G)$ and $G$. In what follows, we establish that, for any $M \subseteq V(G)$, any vertex of $C_{G}(M)$ is close to a vertex of $C_{H}(M)$. Moreover, the diameter of the center of $M$ in $G$ is at most the diameter of the center of $M$ in the injective hull $\mathcal{H}(G)$ plus $2 \alpha(G)$.

Lemma 7. Let $H$ be the injective hull of an $\alpha$-weakly-Helly graph $G$. For any $M \subseteq V(G)$ and integer $\ell \geq 0, C_{G}^{\ell}(M) \subseteq D_{H}\left(C_{H}(M), \alpha+\ell\right)=D_{H}\left(C_{H}^{\ell}(M), \alpha\right)$.

Proof. Consider any vertex $x \in C_{G}^{\ell}(M)$. By Proposition $5, e_{G}^{M}(x) \leq \operatorname{rad}_{G}(M)+\ell \leq \operatorname{rad}_{H}(M)+\alpha+\ell$. By Proposition 3 and Lemma 5 (since $H$ is Helly), $e_{G}^{M}(x)=e_{H}^{M}(x)=\operatorname{rad}_{H}(M)+d_{H}\left(x, C_{H}(M)\right)$. Therefore, $d_{H}\left(x, C_{H}(M)\right) \leq \alpha+\ell$. By Corollary 1, $D_{H}\left(C_{H}(M), \alpha+\ell\right)=D_{H}\left(C_{H}^{\ell}(M), \alpha\right)$.

Theorem 2. Let $H$ be the injective hull of an $\alpha$-weakly-Helly graph $G$. For any integer $\ell \geq 0$ and any $M \subseteq V(G), \operatorname{diam}_{G}\left(C_{G}^{\ell}(M)\right)-2 \alpha \leq \operatorname{diam}_{H}\left(C_{H}^{\ell}(M)\right) \leq \operatorname{diam}_{G}\left(C_{G}^{\alpha+\ell}(M)\right)+2 \alpha$.

Proof. Let $d_{H}(u, v)=\operatorname{diam}_{H}\left(C_{H}^{\ell}(M)\right)$ for some $u, v \in C_{H}^{\ell}(M)$. By Theorem 1, there is a real vertex $u^{*} \in V(G)$ at distance at most $\alpha$ from $u$. By Proposition 3 and Proposition $5, e_{G}^{M}\left(u^{*}\right)=e_{H}^{M}\left(u^{*}\right) \leq$ $e_{H}^{M}(u)+\alpha \leq \operatorname{rad}_{H}(M)+\ell+\alpha \leq \operatorname{rad}_{G}(M)+\ell+\alpha$. Similarly, there is a real vertex $v^{*} \in V(G)$ at distance at most $\alpha$ from $v$ with $e_{G}^{M}\left(v^{*}\right) \leq \operatorname{rad}_{G}(M)+\ell+\alpha$. Both vertices $v^{*}, u^{*}$ belong to $C_{G}^{\alpha+\ell}(M)$. By the triangle inequality, $\operatorname{diam}_{H}\left(C_{H}^{\ell}(M)\right)=d_{H}(u, v) \leq 2 \alpha+d_{H}\left(u^{*}, v^{*}\right) \leq 2 \alpha+\operatorname{diam}_{G}\left(C_{G}^{\alpha+\ell}(M)\right)$.

On the other hand, by Lemma 7 , any vertex of $C_{G}^{\ell}(M)$ has distance at most $\alpha$ to $C_{H}^{\ell}(M)$. Let $x, y \in C_{G}^{\ell}(M)$ such that $d_{G}(x, y)=\operatorname{diam}_{G}\left(C_{G}^{\ell}(M)\right)$. By the triangle inequality, $d_{G}(x, y)=d_{H}(x, y) \leq$ $\operatorname{diam}_{H}\left(C_{H}^{\ell}(M)\right)+2 \alpha$. A small graph depicted on Figure 1 demonstrates that this inequality is tight. 


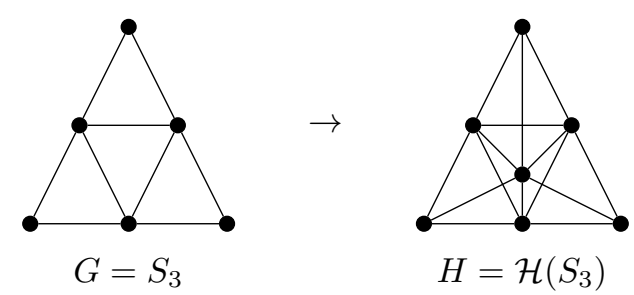

Figure 1: A graph $G$ (left) and its injective hull $H$ (right) which show that inequalities in Proposition 5 and Theorem 2 are tight: $\alpha(G)=1, \operatorname{rad}(G)=2, \operatorname{rad}(H)=1, \operatorname{diam}(C(H))=0, \operatorname{diam}(C(G))=2$.

Corollary 3. For each $\alpha$-weakly-Helly graph $G$ with the injective hull $H=\mathcal{H}(G)$ and every $\ell \geq 0$, $C^{\ell}(H) \cap V(G) \subseteq C^{\ell}(G) \subseteq C^{\ell+\alpha}(H) \cap V(G)$.

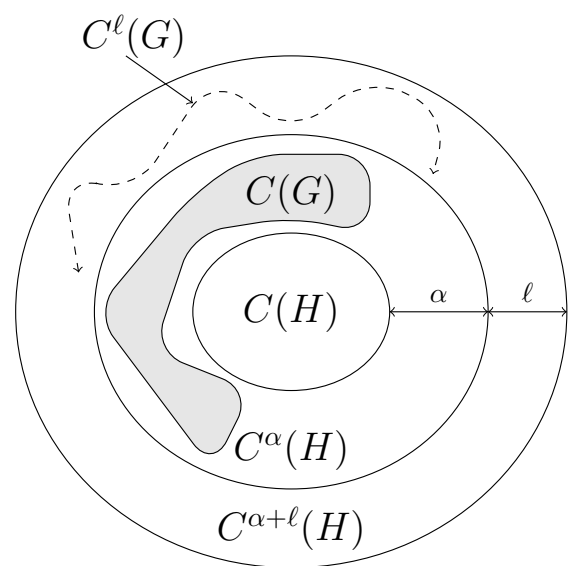

Figure 2: Inclusions for sets $C(G), C(H), C^{\alpha}(H), C^{\ell}(G)$, and $C^{\alpha+\ell}(H)$ in $H=\mathcal{H}(G)$.

Proof. Let $v \in C^{\ell}(H) \cap V(G)$. By Proposition 5 and Proposition 3, $e_{G}(v)=e_{H}(v) \leq \operatorname{rad}(H)+\ell \leq$ $\operatorname{rad}(G)+\ell$. Hence, $v \in C^{\ell}(G)$. Consider now a vertex $v \in C^{\ell}(G)$. It follows from Lemma 7 that $d_{H}(v, C(H)) \leq \ell+\alpha$. By Lemma 15, $e_{H}(v)=\operatorname{rad}(H)+d_{H}(v, C(H)) \leq \operatorname{rad}(H)+\ell+\alpha$. Thus, $v \in C^{\ell+\alpha}(H)$.

The results from this section on inclusions for central sets are illustrated in Figure 2.

\subsection{Relation between Helly-gap and diameter, radius and graph powers}

We obtain a few lower and upper bounds on the Helly-gap $\alpha(G)$. The first follows directly from Proposition 5.

Corollary 4. Let $H:=\mathcal{H}(G)$. For any $M \subseteq V(G), \alpha(G) \geq \operatorname{rad}_{G}(M)-\operatorname{rad}_{H}(M)$.

If $\mathcal{H}(G)$ were given, one could compute $\alpha(G)$ by computing the maximum distance from a Helly vertex to a closest real vertex. However, we provide in Corollary 5 and Lemma 8 upper and lower bounds which do not necessitate computing the injective hull.

Corollary 5. Any graph $G$ is $\alpha$-weakly-Helly for $\alpha \leq\lfloor\operatorname{diam}(G) / 2\rfloor$.

Proof. By contradiction, suppose $\alpha(G)>\lfloor\operatorname{diam}(G) / 2\rfloor$. Let $H:=\mathcal{H}(G)$. By Theorem 1, in $H$ there exists a Helly vertex $u$ with $d_{H}(u, v)>\lfloor\operatorname{diam}(G) / 2\rfloor$ for all $v \in V(G)$. By Proposition 2, $u$ belongs to a shortest $(x, y)$-path for two real vertices $x, y \in V(G)$. As $G$ is isometric in $H, d_{G}(x, y)=d_{H}(x, y)=$ $d_{H}(x, u)+d_{H}(u, y) \geq 2(\lfloor\operatorname{diam}(G) / 2\rfloor+1)>\operatorname{diam}(G)$, a contradiction with $d_{G}(x, y) \leq \operatorname{diam}(G)$. 
Lemma 8. Let $H$ be the injective hull of $G, M$ be any subset of $V(G)$ and $k \geq 0$ be an integer. If $\operatorname{diam}_{G}(M)=2 \operatorname{rad}_{G}(M)-k$, then $\operatorname{rad}_{G}(M)=\operatorname{rad}_{H}(M)+\lfloor k / 2\rfloor$. Moreover, $\alpha(G) \geq\lfloor(2 \operatorname{rad} G(M)-$ $\left.\left.\operatorname{diam}_{G}(M)\right) / 2\right\rfloor$, that is, $2 \operatorname{rad}_{G}(M) \geq \operatorname{diam}_{G}(M) \geq 2 \operatorname{rad}_{G}(M)-2 \alpha(G)-1$.

Proof. By Proposition 4, $2 \operatorname{rad}_{G}(M)-k=\operatorname{diam}_{G}(M)=\operatorname{diam}_{H}(M)$. Since $H$ is Helly, by Lemma 4 , $\operatorname{rad}_{H}(M)=\left\lceil\left(2 \operatorname{rad}_{G}(M)-k\right) / 2\right\rceil=\operatorname{rad}_{G}(M)-\lfloor k / 2\rfloor$. By Corollary $4, \alpha(G) \geq\lfloor k / 2\rfloor$.

Remark. Observe that the Helly-gap of a graph $G$ can be much larger than $\operatorname{rad}(G)-\operatorname{rad}(H)$ and $\lfloor(2 \operatorname{rad}(G)-\operatorname{diam}(G)) / 2\rfloor$. Consider a graph $G$ formed by a cycle $C_{4 k}$ of size $4 k$ and two paths of length $k$ each connected to opposite ends of the cycle, as illustrated in Fig. 3. By Lemma $8, \alpha\left(C_{4 k}\right) \geq k$. By Corollary 2, $\alpha(G)=\alpha\left(C_{4 k}\right) \geq k$. However, $\operatorname{diam}(G)=4 k$ and $\operatorname{rad}(G)=2 k$, and by Lemma 8 , $\operatorname{rad}(G)=\operatorname{rad}(H)$. In this case, $\operatorname{rad}(G)-\operatorname{rad}(H)=\lfloor(2 \operatorname{rad}(G)-\operatorname{diam}(G)) / 2\rfloor=0$.

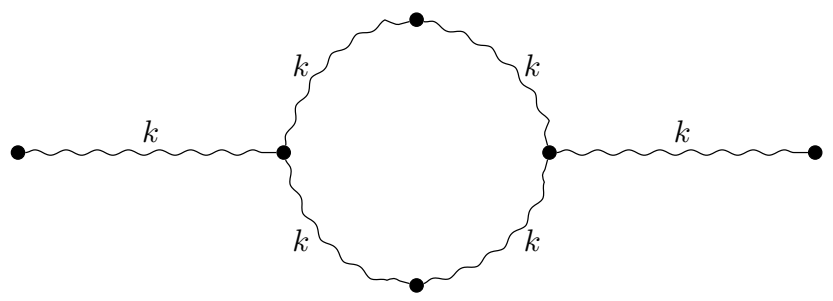

Figure 3: A graph $G$ with Helly-gap $k$ and $\operatorname{rad}(G)=\operatorname{rad}(H)=2 k$.

Corollary 6. Let $H$ be the injective hull of $G$ and $M \subseteq V(G)$. Then, $\operatorname{diam}_{G}(M) \geq 2 \operatorname{rad}_{G}(M)-1$ if and only if $C_{G}(M) \subseteq C_{H}(M)$.

Proof. If $\operatorname{diam}_{G}(M) \geq 2 \operatorname{rad}_{G}(M)-1$, then by Lemma 8, $\operatorname{rad}_{G}(M)=\operatorname{rad}_{H}(M)$. Thus, any $v \in C_{G}(M)$ has $e_{H}^{M}(v) \leq \operatorname{rad}_{H}(M)$. On the other hand, if $C_{G}(M) \subseteq C_{H}(M)$, since eccentricities are preserved in $H$ by Proposition 3 , then also $\operatorname{rad}_{G}(M)=\operatorname{rad}_{H}(M)$. Since $H$ is Helly, by Lemma 4 and Proposition 4 , $\operatorname{diam}_{G}(M)=\operatorname{diam}_{H}(M) \geq 2 \operatorname{rad}_{H}(M)-1=2 \operatorname{rad}_{G}(M)-1$ holds.

Interestingly, the Helly-gap of a graph $G$ decreases in powers of $G$.

Lemma 9. Let $G$ be an $\alpha$-weakly-Helly graph. For every integer $k \geq 1, G^{k}$ is $\lceil\alpha / k\rceil$-weakly-Helly.

Proof. Let $\mathcal{F}=\left\{D_{G^{k}}(v, r(v)): v \in S\right\}$ be a system of disks which pairwise intersect in $G^{k}$ so that any two vertices $u, v \in S$ satisfy $d_{G^{k}}(u, v) \leq r(u)+r(v)$. Then, their distances in $G$ satisfy $d_{G}(u, v) \leq k(r(u)+r(v))$. Consider now a corresponding system of disks in $G$ centered at same vertices, defined as $\mathcal{M}=\left\{D_{G}(v, k r(v)): v \in S\right\}$. $\mathcal{M}$ is a family of pairwise intersecting disks of $G$ as any two vertices $u, v \in S$ satisfy $d_{G}(u, v) \leq k r(u)+k r(v)$. As $G$ is $\alpha$-weakly-Helly, there exists a common vertex $z \in \cap\left\{D_{G}(v, k r(v)+\alpha): v \in S\right\}$. Since, for any $v \in S, d_{G}(z, v) \leq k r(v)+\alpha$, necessarily, $d_{G^{k}}(z, v) \leq r(v)+\lceil\alpha / k\rceil$. Hence, vertex $z$ intersects all disks of $\mathcal{F}$ when the radii of each disk is extended by $\lceil\alpha / k\rceil$. Therefore, $G^{k}$ is $\lceil\alpha / k\rceil$-weakly-Helly.

The results of this subsection are summarized in Theorem 3 ,

Theorem 3. Let $G$ be an arbitrary graph. Then, the following holds:

i) $\lfloor(2 \operatorname{rad}(G)-\operatorname{diam}(G)) / 2\rfloor \leq \alpha(G) \leq\lfloor\operatorname{diam}(G) / 2\rfloor$, and

ii) $\alpha\left(G^{k}\right) \leq\lceil\alpha(G) / k\rceil$.

This Theorem 3 generalizes some known results for Helly graphs. Recall that, in Helly graphs, $\lfloor(2 \operatorname{rad}(G)-\operatorname{diam}(G)) / 2\rfloor=0$ holds and that every power of a Helly graph is a Helly graph as well [16]. We know also (see also Section 6) that the Helly-gap of a chordal graph or a distance-hereditary graph is at most 1 and the Helly-gap of a $\delta$-hyperbolic graph is at most $2 \delta$. Hence, Theorem 3 generalizes some known results on those graphs, too. For every chordal graph $G$ as well as for every distancehereditary graph $G,\lfloor(2 \operatorname{rad}(G)-\operatorname{diam}(G)) / 2\rfloor \leq 1$ holds [9, 18, 51]. For every $\delta$-hyperbolic graph $G$, $\lfloor(2 \operatorname{rad}(G)-\operatorname{diam}(G)) / 2\rfloor \leq 2 \delta$ holds [11,24]. 


\subsection{The eccentricity function is almost unimodal in $\alpha$-weakly-Helly graphs}

The locality $\operatorname{loc}(v)$ of a vertex $v$ is defined as the minimum distance from $v$ to a vertex of strictly smaller eccentricity. Recall that the eccentricity function $e_{G}(\cdot)$ is unimodal in $G$ if every vertex $v \in V(G) \backslash C(G)$ has a neighbor $u$ such that $e_{G}(u)<e_{G}(v)$, i.e., $\operatorname{loc}(v)=1$. In a graph $G$ with a unimodal eccentricity function, any local minimum of the eccentricity function (i.e., a vertex whose eccentricity is not larger than the eccentricity of any of its neighbors) is the global minimum of the eccentricity function in $G$ (i.e., it is a central vertex). Recall that Helly graphs are characterized by the property that every eccentricity function $e_{G}^{M}$ is unimodal for any $M \subseteq V(G)$; therefore, $e_{G}^{M}(v)=d\left(v, C_{G}(M)\right)+\operatorname{rad}_{G}(M)$ holds (see Lemma [5). A natural question for $\alpha$-weakly-Helly graphs is whether similar results on the unimodality of the eccentricity function hold up to a function of $\alpha$, that is, if any vertex $v \in$ $V(G) \backslash C^{\alpha}(G)$ has $l o c(v) \leq f(\alpha)$. The following lemmas answer in the positive.

Lemma 10. Let $G$ be an $\alpha$-weakly-Helly graph and let $M \subseteq V(G)$. If there is a vertex $v \in V(G)$ such that $e_{G}^{M}(v)>\operatorname{rad}_{G}(M)+\alpha$, then there is a vertex $u \in D_{G}(v, 2 \alpha+1)$ with $e_{G}^{M}(u)<e_{G}^{M}(v)$.

Proof. Let $S=M \cup\{v\}$. Consider in $G$ a system of disks $\mathcal{F}=\left\{D\left(u, \rho_{u}\right): u \in S\right\}$, where the radii are defined as $\rho_{w}=e_{G}^{M}(v)-1-\alpha$ for any $w \in M$, and $\rho_{v}=\alpha+1$. We assert that all disks of $\mathcal{F}$ are pairwise intersecting. Clearly for any $w \in M$, disks $D\left(v, \rho_{v}\right)$ and $D\left(w, \rho_{w}\right)$ intersect as $d(w, v) \leq e_{G}^{M}(v)$. We now show that for any $w, w^{\prime} \in M$ the disks $D\left(w, \rho_{w}\right)$ and $D\left(w^{\prime}, \rho_{w^{\prime}}\right)$ intersect.

Consider a vertex $c \in C_{G}(M)$. By choice of $v, e_{G}^{M}(v) \geq \operatorname{rad}_{G}(M)+\alpha+1=e_{G}^{M}(c)+\alpha+1$. By the triangle inequality, for any two vertices $w, w^{\prime} \in M$ we have $d\left(w, w^{\prime}\right) \leq d(w, c)+d\left(w^{\prime}, c\right) \leq$ $e_{G}^{M}(c)+e_{G}^{M}(c) \leq\left(e_{G}^{M}(v)-1-\alpha\right)+\left(e_{G}^{M}(v)-1-\alpha\right)=\rho_{w}+\rho_{w^{\prime}}$. Then, by the $\alpha$-weakly-Helly property, the system $\mathcal{F}$ of pairwise intersecting disks has a common intersection when radii of all disks are extended by $\alpha$. Therefore, there is a vertex $u$ such that $d(u, v) \leq 2 \alpha+1$ and $d(u, w) \leq e_{G}^{M}(v)-1$ for all $w \in M$.

For $\alpha=0$ we obtain a result known for Helly graphs. As $\delta$-hyperbolic graphs are $(2 \delta)$-weakly-Helly (this follows from a result in [14, see also Section [6), Lemma 10 also greatly generalizes a result known for $\delta$-hyperbolic graphs (see [23]).

Lemma 11. Let $H$ be the injective hull of an $\alpha$-weakly-Helly graph $G$, and let $M \subseteq V(G)$. If there is a vertex $v \in V(G)$ such that $d_{H}\left(v, C_{H}(M)\right)>\alpha$, then there is a real vertex $u \in D_{G}(v, 2 \alpha+1)$ such that $e_{G}^{M}(u)<e_{G}^{M}(v)$.

Proof. Let $c \in C_{H}(M)$ be a vertex closest in $H$ to $v$, and assume that $d_{H}(v, c) \geq \alpha+1$. Let $w \in V(H)$ be a vertex on a shortest $(v, c)$-path of $H$ such that $d_{H}(v, w)=\alpha+1$. Since $H$ is Helly, by Proposition 3 and Lemma 5, $e_{G}^{M}(v)=e_{H}^{M}(v)=d_{H}\left(v, C_{H}(M)\right)+\operatorname{rad}_{H}(M)=d_{H}(v, w)+d_{H}(w, c)+\operatorname{rad}_{H}(M)=$ $\alpha+1+e_{H}^{M}(w)$. Therefore, $e_{H}^{M}(w)=e_{G}^{M}(v)-\alpha-1$. Since $G$ is $\alpha$-weakly-Helly, by Theorem 1, there is a vertex $u \in V(G)$ such that $d_{H}(u, w) \leq \alpha$. Hence, $e_{G}^{M}(u)=e_{H}^{M}(u) \leq e_{H}^{M}(w)+\alpha \leq e_{G}^{M}(v)-1$. See Fig. 4 for an illustration.

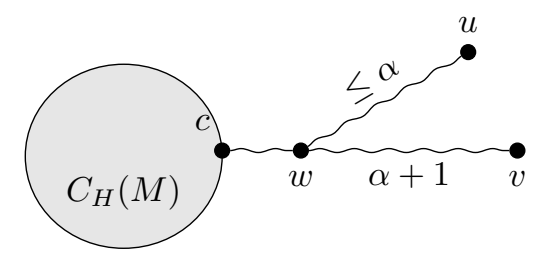

Figure 4: Illustration to the proof of Lemma 11.

Theorem 4 summarizes the results of Lemma 10 and Lemma 11.

Theorem 4. Let $H$ be the injective hull of an $\alpha$-weakly-Helly graph $G$, and let $M \subseteq V(G)$. If there is a vertex $v \in V(G)$ such that $e_{G}^{M}(v)>\operatorname{rad}_{G}(M)+\alpha$ or $d_{H}\left(v, C_{H}(M)\right)>\alpha$, then there is a real vertex $u \in D_{G}(v, 2 \alpha+1)$ such that $e_{G}^{M}(u)<e_{G}^{M}(v)$. 


\section{$5 \quad$ Estimating all eccentricities}

This section provides upper and lower bounds on the eccentricity of a vertex $v$ based on a variety of conditions: the distance from $v$ to a closest almost central vertex (i.e., a closest vertex with eccentricity at most $\operatorname{rad}_{G}(M)+\alpha$ ) and whether $v$ is a farthest vertex from some other vertex and if $\operatorname{diam}\left(C_{G}^{2 \alpha}(M)\right)$ is bounded. We also prove that any $\alpha$-weakly-Helly graph has an eccentricity approximating spanning tree where the additive approximation error depends on the diameter of the $\operatorname{set} C^{\alpha}(G)$. Finally, we describe the eccentricity terrain in $\alpha$-weakly-Helly graphs, that is, how vertex eccentricities change along vertices of a shortest path to $C_{G}^{\alpha}(M)$.

\subsection{Using distances to $C_{G}^{\alpha}(M)$}

Lemma 12. Let $G$ be a graph, $M \subseteq V(G)$, and $k \geq 0$. For every vertex $x \in V(G)$, $e_{G}^{M}(x) \leq$ $d\left(x, C_{G}^{k}(M)\right)+\operatorname{rad}_{G}(M)+k$ holds.

Proof. Let $e_{G}^{M}(x)=d(x, y)$ where $y \in F_{G}^{M}(x)$, and let $x_{p} \in C_{G}^{k}(M)$ be closest to $x$. By the triangle inequality, $e_{G}^{M}(x) \leq d\left(x, x_{p}\right)+d\left(x_{p}, y\right) \leq d\left(x, C_{G}^{k}(M)\right)+\operatorname{rad}_{G}(M)+k$.

Lemma 13. Let $G$ be an $\alpha$-weakly-Helly graph and $M \subseteq V(G)$. For every vertex $x \in V(G), e_{G}^{M}(x) \geq$ $d\left(x, C_{G}^{\alpha}(M)\right)+\operatorname{rad}_{G}(M)-\alpha$ holds.

Proof. Let $H:=\mathcal{H}(G)$ and $h \in C_{H}(M)$ be a closest vertex to $x$ in $H$. By Lemma 5 and Proposition 3 , $d_{H}(x, h)=d_{H}\left(x, C_{H}(M)\right)=e_{H}^{M}(x)-\operatorname{rad}_{H}(M)=e_{G}^{M}(x)-\operatorname{rad}_{H}(M) \geq \operatorname{rad}_{G}(M)-\operatorname{rad}_{H}(M)$. Then, let $y$ be a vertex on a shortest $(x, h)$-path with $d_{H}(h, y)=\operatorname{rad}_{G}(M)-\operatorname{rad}_{H}(M)$. By Lemma 5 , $e_{H}^{M}(y)=d_{H}\left(y, C_{H}(M)\right)+\operatorname{rad}_{H}(M)=d_{H}(y, h)+\operatorname{rad}_{H}(M)=\operatorname{rad}_{G}(M)$. By Theorem 1, there is a real vertex $y^{*} \in V(G)$ such that $d_{H}\left(y, y^{*}\right) \leq \alpha$. Applying Proposition 3 one obtains $e_{G}^{M}\left(y^{*}\right)=e_{H}^{M}\left(y^{*}\right) \leq$ $\operatorname{rad}_{G}(M)+\alpha$. By the triangle inequality, $d_{H}(x, y) \geq d_{H}\left(x, y^{*}\right)-d_{H}\left(y, y^{*}\right) \geq d\left(x, C_{G}^{\alpha}(M)\right)-\alpha$. Thus, $e_{G}^{M}(x)=d_{H}(x, h)+\operatorname{rad}_{H}(M)=d_{H}(x, y)+\operatorname{rad}_{G}(M) \geq d\left(x, C_{G}^{\alpha}(M)\right)+\operatorname{rad}_{G}(M)-\alpha$.

Applying Lemma 12 with $k=\alpha$ and Lemma 13, we obtain the following approximation of eccentricities in $\alpha$-weakly-Helly graphs.

Theorem 5. Let $G$ be an $\alpha$-weakly-Helly graph. For every $M \subseteq V(G)$, every $x \in V(G)$ satisfies

$$
d\left(x, C_{G}^{\alpha}(M)\right)+\operatorname{rad}_{G}(M)-\alpha \leq e_{G}^{M}(x) \leq d\left(x, C_{G}^{\alpha}(M)\right)+\operatorname{rad}_{G}(M)+\alpha .
$$

If $C_{G}^{\alpha}(M)$ is known in advance, then one obtains a linear time additive $2 \alpha$-approximation of all eccentricities by using a breadth-first search from $C_{G}^{\alpha}(M)\left(B F S\left(C_{G}^{\alpha}(M)\right)\right)$, to obtain all distances to $C_{G}^{\alpha}(M)$, and a $B F S(c)$ from any fixed $c \in C_{G}^{\alpha}(M)$ that has a neighbor not in $C_{G}^{\alpha}(M)$, to compute $\operatorname{rad}_{G}(M)+\alpha$. Then, for each vertex $v$, set an approximate eccentricity $\hat{e}^{M}(v)=d_{G}\left(v, C_{G}^{\alpha}(M)\right)+$ $\operatorname{rad}_{G}(M)+\alpha$. By Theorem 5 , $e_{G}^{M}(v) \leq \hat{e}^{M}(v) \leq e_{G}^{M}(v)+2 \alpha$.

By Corollary 1, any Helly graph $G$ satisfies $C_{G}^{\ell}(M)=D\left(C_{G}(M), \ell\right)$. This also extends to $\alpha$-weaklyHelly graphs in the following way.

Corollary 7. Let $G$ be an $\alpha$-weakly-Helly graph. For any $M \subseteq V(G)$ and any integer $\ell \geq 0, C_{G}^{\ell}(M) \subseteq$ $D_{G}\left(C_{G}^{\alpha}(M), \alpha+\ell\right)$.

Proof. If $x \in C_{G}^{\ell}(M)$ then, by Theorem 5, $d\left(x, C_{G}^{\alpha}(M)\right)+\operatorname{rad}_{G}(M)-\alpha \leq e_{G}^{M}(x) \leq \ell+\operatorname{rad}_{G}(M)$. Hence, $d\left(x, C_{G}^{\alpha}(M)\right) \leq \alpha+\ell$.

We can restate the lower bound on $e_{G}^{M}(x)$ in Theorem [5 by using thinnes $\kappa(\mathcal{H}(G))$ of metric intervals of a graph's injective hull. 
Lemma 14. Let $H$ be the injective hull of a graph $G$. For any $M \subseteq V(G)$, every $x \in V(G)$ satisfies $e_{G}^{M}(x) \geq d_{G}\left(x, C_{G}^{\kappa(H)}(M)\right)+\operatorname{rad}_{G}(M)$.

Proof. We apply the same idea as in the proof of Lemma 13 with vertex $x$, where $e_{G}^{M}(x)=e_{H}^{M}(x)=$ $d_{H}(x, t)$ for some vertex $t \in M$. Let $h \in C_{H}(M)$ be a closest vertex to $x$, and let $y$ be a vertex on a shortest $(x, h)$-path with $d_{H}(h, y)=\operatorname{rad}_{G}(M)-\operatorname{rad}_{H}(M)$ and $e_{H}^{M}(y)=\operatorname{rad}_{G}(M)$. Then, since $G$ is isometric in $H$, there is a shortest $(x, t)$-path $P$ in $H$ such that each vertex $v \in P$ belongs to $V(G)$. Note also that, by Lemma 5, $h$ is on a shortest path from $x$ to $t$ in $H$. Let $y^{*} \in P$ such that $d_{G}\left(y^{*}, t\right)=\operatorname{rad}_{G}(M)$ so that $y$ and $y^{*}$ belong to the same interval slice $S_{r a d_{G}(M)}(t, x)$ in $H$. Therefore, $d_{H}\left(y, y^{*}\right) \leq \kappa(H)$ and, by Proposition 3 , $e_{G}^{M}\left(y^{*}\right)=e_{H}^{M}\left(y^{*}\right) \leq e_{H}^{M}(y)+\kappa(H)=\operatorname{rad}_{G}(M)+\kappa(H)$. Hence, $e_{G}^{M}(x)=d_{H}(x, y)+\operatorname{rad}_{G}(M)=d_{H}\left(x, y^{*}\right)+\operatorname{rad}_{G}(M) \geq d_{H}\left(x, C_{G}^{\kappa(H)}(M)\right)+\operatorname{rad}_{G}(M)$ as $y^{*} \in C_{G}^{\kappa(H)}(M)$.

As in a $\delta$-hyperbolic graph $G, \kappa(\mathcal{H}(G)) \leq 2 \delta$ (see Subsection 6.2), Lemma 14 generalizes a known result for $\delta$-hyperbolic graphs: $e_{G}(x) \geq d_{G}\left(x, C^{2 \delta}(G)\right)+\operatorname{rad}(G)$ for all $x \in V(G)$ [23]. Note that similar results are known for chordal graphs: $e_{G}(x) \geq d_{G}(x, C(G))+\operatorname{rad}(G)-1$ for every $x \in V(G)$ [26], and for distance-hereditary graphs: $e_{G}(x)=d_{G}\left(x, C^{1}(G)\right)+\operatorname{rad}(G)+1$ for every $x \in V(G) \backslash C(G)$ [22].

\subsection{A vertex furthest from some other vertex}

Recall that in a Helly graph $G$, for every vertex $x \in V$ and every farthest vertex $y \in F(x), e(y) \geq$ $2 \operatorname{rad}(G)-\operatorname{diam}(C(G))$ holds $[16$.

Theorem 6. Let $G$ be an $\alpha$-weakly-Helly graph. Then, for every $M \subseteq V(G)$ and every $x \in V(G)$, each vertex $y \in F_{G}^{M}(x)$ satisfies

$$
\begin{gathered}
e_{G}^{M}(y) \geq \operatorname{rad}_{G}(M)-\operatorname{diam}_{G}\left(C_{G}^{2 \alpha}(M)\right)-2 \alpha, \\
e_{G}^{M}(y) \geq \operatorname{rad}_{G}(M)-\operatorname{diam}_{G}\left(C_{G}^{\alpha}(M)\right)-4 \alpha .
\end{gathered}
$$

Proof. Let $H:=\mathcal{H}(G)$ and let $e_{G}^{M}(y)=d_{G}(y, w)$ for some $w \in M$. As $e_{H}^{M}(\cdot)$ is unimodal, in $H$ there is a closest to $x$ vertex $b_{h} \in C_{H}(M)$ such that $e_{G}^{M}(x)=d_{H}\left(x, b_{h}\right)+\operatorname{rad}_{H}(M)$. Similarly, in $H$ there is a closest to $y$ vertex $c_{h} \in C_{H}(M)$ such that $e_{G}^{M}(y)=d_{H}\left(y, c_{h}\right)+\operatorname{rad}_{H}(M)$. Then, $d_{H}\left(x, C_{H}(M)\right)=$ $d_{H}\left(x, b_{h}\right)=e_{H}^{M}(x)-\operatorname{rad}_{H}(M) \geq \operatorname{rad}_{G}(M)-\operatorname{rad}_{H}(M)$ and, by symmetry, $d_{H}\left(y, c_{h}\right) \geq \operatorname{rad}_{G}(M)-$ $\operatorname{rad}_{H}(M)$. Now let $c \in I\left(c_{h}, y\right)$ be a vertex in $H$ such that $d_{H}\left(c_{h}, c\right)=\operatorname{rad}_{G}(M)-\operatorname{rad}_{H}(M)$, and also $b \in I\left(b_{h}, x\right)$ be a vertex in $H$ such that $d_{H}\left(b_{h}, b\right)=\operatorname{rad}_{G}(M)-\operatorname{rad}_{H}(M)$, as illustrated in Fig. 5. By Proposition 5, $e_{H}^{M}(b) \leq \operatorname{rad}_{G}(M)-\operatorname{rad}_{H}(M)+e_{H}^{M}\left(b_{h}\right)=\operatorname{rad}_{G}(M) \leq \operatorname{rad}_{H}(M)+\alpha$. By symmetry, $e_{H}^{M}(c) \leq \operatorname{rad}_{H}(M)+\alpha$, and so both $b$ and $c$ belong to $C_{H}^{\alpha}(M)$. By the triangle inequality, $\operatorname{rad}_{G}(M)=d_{H}(b, y) \leq d_{H}(b, c)+d_{H}(c, y)=d_{H}(b, c)+d_{H}(w, y)-\operatorname{rad}_{G}(M)$. That is, $e_{G}^{M}(y)=d_{H}(w, y) \geq 2 \operatorname{rad}_{G}(M)-d_{H}(b, c) \geq 2 \operatorname{rad}_{G}(M)-\operatorname{diam}_{H}\left(C_{H}^{\alpha}(M)\right)$. By Corollary 1, as $H$ is Helly, $e_{G}^{M}(y) \geq 2 \operatorname{rad}_{G}(M)-\operatorname{diam}_{H}\left(C_{H}^{\alpha}(M)\right)=2 \operatorname{rad}_{G}(M)-\operatorname{diam}_{H}\left(C_{H}(M)\right)-2 \alpha$. Applying now Theorem 2 with $\ell=\alpha$, we get $\operatorname{diam}_{H}\left(C_{H}^{\alpha}(M)\right) \leq 2 \alpha+\operatorname{diam}_{G}\left(C_{G}^{2 \alpha}(M)\right)$ and hence $e_{G}^{M}(y) \geq$ $2 \operatorname{rad}_{G}(M)-\operatorname{diam}_{G}\left(C_{G}^{2 \alpha}(M)\right)-2 \alpha$. Applying Theorem 2 with $\ell=0$, we get $\operatorname{diam}_{H}\left(C_{H}(M)\right) \leq$ $2 \alpha+\operatorname{diam}_{G}\left(C_{G}^{\alpha}(M)\right)$ and hence $e_{G}^{M}(y) \geq 2 \operatorname{rad}_{G}(M)-\operatorname{diam}_{G}\left(C_{G}^{\alpha}(M)\right)-4 \alpha$.

It is known that for every vertex $x$, any vertex $y \in F_{G}(x)$ satisfies $e_{G}(y) \geq 2 \operatorname{rad}(G)-3$ if $G$ is a chordal graph or a distance-hereditary graph [12,18] and $e_{G}(y) \geq \operatorname{diam}(G)-2 \delta \geq 2 \operatorname{rad}(G)-6 \delta-1$ if $G$ is a $\delta$-hyperbolic graph [11,24. Furthermore, for every chordal or distance-hereditary graph $G$, $\operatorname{diam}_{G}(C(G)) \leq 3$ holds [9, 50, 51] and for every $\delta$-hyperbolic graph $G$, $\operatorname{diam}_{G}\left(C^{2 \delta}(G)\right) \leq 8 \delta+1$ and $\operatorname{diam}_{G}(C(G)) \leq 4 \delta+1$ hold [11,24]. Recall that the diameter of the center of a Helly graph cannot be bounded. Although $C(G)$ itself is Helly and is isometric in $G$ for a Helly graph $G$ [16], $C(G)$ can have an arbitrarily large diameter as any Helly graph is the center of some Helly graph [16]. Thus, $\operatorname{diam}_{G}\left(C^{\alpha(G)}(G)\right)$ cannot be bounded by a function of $\alpha(G)$ (even for $\alpha(G)=0$, i.e., for Helly graphs). 


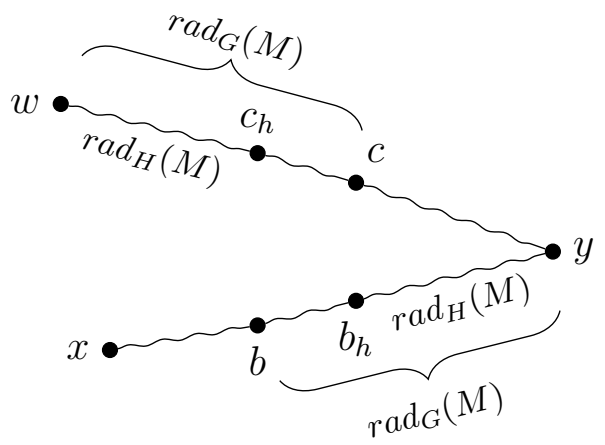

Figure 5: Illustration to the proof of Theorem 6.

\subsection{Eccentricity approximating spanning tree}

An eccentricity $t$-approximating spanning tree is a spanning tree $T$ such that $e_{T}(v)-e_{G}(v) \leq t$ holds for all $v \in V(G)$. Such a tree tries to approximately preserve only distances from $v$ to its farthest vertices, allowing for a larger increase in distances to nearby vertices. Many classes of graphs have an eccentricity $t$-approximating spanning tree, where $t$ is a small constant (e.g., see [13, 23, 26, 43, 45] and papers cited therein). We will show that $\alpha$-weakly-Helly graphs have an eccentricity $t$-approximating spanning tree where $t$ depends linearly on $\alpha$ and on the diameter of $C^{\alpha}(G)$.

Lemma 15. Let $H$ be the injective hull of an $\alpha$-weakly-Helly graph $G$. For every $M \subseteq V(G)$, there is a real vertex $c^{*} \in V(G)$ such that, for every vertex $x \in V(G), d_{G}\left(x, c^{*}\right)+e_{G}^{M}\left(c^{*}\right)-e_{G}^{M}(x) \leq$ $\left\lceil\frac{\operatorname{diam}_{H}\left(C_{H}(M)\right)}{2}\right\rceil+2 \alpha$ holds.

Proof. Let $c \in V(H)$ be a vertex belonging to $C_{H}\left(C_{H}(M)\right)$. By Theorem 1, there is a real vertex $c^{*} \in V(G)$ such that $d_{H}\left(c, c^{*}\right) \leq \alpha$.

We first establish a few useful inequalities. Since $H$ is Helly, by Lemma 1, $C_{H}(M)$ and $C_{H}\left(C_{H}(M)\right)$ are also Helly and isometric in $H$. Thus, by Lemma 4 $\operatorname{rad}_{H}(M)=\left\lceil\operatorname{diam}_{H}(M) / 2\right\rceil$ and $\operatorname{rad}_{H}\left(C_{H}(M)\right)=\left\lceil\operatorname{diam}_{H}\left(C_{H}(M)\right) / 2\right\rceil$. Let $c_{x} \in C_{H}(M)$ be a closest vertex to $x$. By Lemma 5 , $e_{G}^{M}(x)=e_{H}^{M}(x)=d_{H}\left(x, C_{H}(M)\right)+\operatorname{rad}_{H}(M)=d_{H}\left(x, c_{x}\right)+\left\lceil\operatorname{diam}_{H}(M) / 2\right\rceil$. By the triangle inequality, $e_{G}^{M}\left(c^{*}\right)=e_{H}^{M}\left(c^{*}\right) \leq e_{H}^{M}(c)+\alpha=\left\lceil\operatorname{diam}_{H}(M) / 2\right\rceil+\alpha$. Since $G$ is isometric in $H, d_{G}\left(x, c^{*}\right)=d_{H}\left(x, c^{*}\right) \leq$ $d_{H}\left(x, c_{x}\right)+d_{H}\left(c_{x}, c\right)+d_{H}\left(c, c^{*}\right) \leq d_{H}\left(x, c_{x}\right)+\operatorname{rad}_{H}\left(C_{H}(M)\right)+\alpha=d_{H}\left(x, c_{x}\right)+\left\lceil\operatorname{diam}_{H}\left(C_{H}(M)\right) / 2\right\rceil+\alpha$. We are now ready to prove the claim.

$$
\begin{aligned}
e_{G}^{M}(x)=e_{H}^{M}(x) & =d_{H}\left(x, c_{x}\right)+\left\lceil\operatorname{diam}_{H}(M) / 2\right\rceil \\
& \geq\left(d_{G}\left(x, c^{*}\right)-\left\lceil\operatorname{diam}_{H}\left(C_{H}(M)\right) / 2\right\rceil-\alpha\right)+\left\lceil\operatorname{diam}_{H}(M) / 2\right\rceil \\
& \geq d_{G}\left(x, c^{*}\right)-\left\lceil\operatorname{diam}_{H}\left(C_{H}(M)\right) / 2\right\rceil+e_{G}^{M}\left(c^{*}\right)-2 \alpha .
\end{aligned}
$$

Therefore, $\left.d_{G}\left(x, c^{*}\right)+e_{G}^{M}\left(c^{*}\right)-e_{G}^{M}(x) \leq \operatorname{diam}_{H}\left(C_{H}(M)\right) / 2\right\rceil+2 \alpha$.

Theorem 7. Let $G$ be an $\alpha$-weakly-Helly graph. For every $M \subseteq V(G), G$ has a spanning tree $T$ such that, for all $x \in V(G), e_{T}^{M}(x)-e_{G}^{M}(x) \leq\left\lceil\operatorname{diam}_{G}\left(C_{G}^{\alpha}(M)\right) / 2\right\rceil+3 \alpha$.

Proof. By Lemma 15, there exists a vertex $c^{*} \in V(G)$ such that any vertex $x \in V(G)$ satisfies $d_{G}\left(x, c^{*}\right)+e_{G}^{M}\left(c^{*}\right)-e_{G}^{M}(x) \leq\left\lceil\operatorname{diam}_{H}\left(C_{H}(M)\right) / 2\right\rceil+2 \alpha$. Let $T$ be a BFS tree of $G$ rooted at $c^{*}$. Then, any vertex $x \in V(G)$ has $e_{T}^{M}(x)-e_{G}^{M}(x) \leq d_{T}\left(x, c^{*}\right)+e_{T}^{M}\left(c^{*}\right)-e_{G}^{M}(x)$. As distances to $c^{*}$ are preserved in $T$, one obtains $e_{T}^{M}(x)-e_{G}^{M}(x) \leq\left\lceil\operatorname{diam}_{H}\left(C_{H}(M)\right) / 2\right\rceil+2 \alpha$. Applying Theorem 2 with $\ell=0$, $\operatorname{diam}_{H}\left(C_{H}(M)\right) \leq \operatorname{diam}_{G}\left(C_{G}^{\alpha}(M)\right)+2 \alpha$. Hence, $\left.e_{T}^{M}(x)-e_{G}^{M}(x) \leq\left\lceil\left(\operatorname{diam}_{G}\left(C_{G}^{\alpha}(M)\right)+2 \alpha\right) / 2\right)\right\rceil+2 \alpha$, establishing the desired result. 
It is known that every chordal graph and every distance-hereditary graph has an eccentricity 2approximating spanning tree [19,26, 39, 45] and every $\delta$-hyperbolic graph has an eccentricity $(4 \delta+1)-$ approximating spanning tree [23. These graph classes have additional nice properties that allowed to get for them sharper results than the one of Theorem 7 which holds for general $\alpha$-weakly-Helly graphs.

\subsection{Eccentricity terrain}

We can describe the behavior of the eccentricity function in a graph in terms of graph's eccentricity terrain, that is, how vertex eccentricities change along vertices of a shortest path to $C_{G}^{\alpha}(M)$. We define an ordered pair of vertices $(u, v)$, where $(u, v) \in E$, as an up-edge if $e_{G}^{M}(u)<e_{G}^{M}(v)$, as a downedge if $e_{G}^{M}(u)>e_{G}^{M}(v)$, and as a horizontal-edge if $e_{G}^{M}(u)=e_{G}^{M}(v)$. Let $\mathbb{U}(P(y, x)), \mathbb{H}(P(y, x))$, and $\mathbb{D}(P(y, x))$ denote the number of up-edges, horizontal-edges, and down-edges along a shortest path $P(y, x)$ from $y \in V(G)$ to $x \in V(G)$.

We note that Lemma 16 is shown in 23 for the eccentricity function $e_{G}(\cdot)$. For completeness, we provide a proof that it holds for $e_{G}^{M}(\cdot)$ for any $M \subseteq V(G)$.

Lemma 16. [23] Let $G$ be an arbitrary graph and $M \subseteq V(G)$. For any shortest path $P(y, x)$ of $G$ from a vertex $y$ to a vertex $x$ the following holds:

i) $\mathbb{D}(P(y, x))-\mathbb{U}(P(y, x))=e_{G}^{M}(y)-e_{G}^{M}(x)$, and

ii) $2 \mathbb{U}(P(y, x))+\mathbb{H}(P(y, x))=d_{G}(y, x)-\left(e_{G}^{M}(y)-e_{G}^{M}(x)\right)$.

Proof. We use an induction on $d_{G}(y, v)$ for any vertex $v \in P(y, x)$. First, assume that $v$ is adjacent to $y$. If $(y, v)$ is an up-edge, then $e_{G}^{M}(y)-e_{G}^{M}(v)=-1$ and $\mathbb{D}(P(y, v))-\mathbb{U}(P(y, v))=-1$. If $(y, v)$ is a horizontal-edge, then $e_{G}^{M}(y)-e_{G}^{M}(v)=0$ and $\mathbb{D}(P(y, v))-\mathbb{U}(P(y, v))=0$. If $(y, v)$ is a down-edge, then $e_{G}^{M}(y)-e_{G}^{M}(v)=1$ and $\mathbb{D}(P(y, v))-\mathbb{U}(P(y, v))=1$. Now consider an arbitrary vertex $v \in P(y, x)$ and assume, by induction, that $e_{G}^{M}(y)-e_{G}^{M}(v)=\mathbb{D}(P(y, v))-\mathbb{U}(P(y, v))$. Let vertex $u \in P(y, x)$ be adjacent to $v$ with $d(y, u)=d(y, v)+1$. By definition, $\mathbb{D}(P(y, u))=\mathbb{D}(P(y, v))+\mathbb{D}((v, u))$ and $\mathbb{U}(P(y, u))=\mathbb{U}(P(y, v))+\mathbb{U}((v, u))$. We consider three cases based on the classification of edge $(v, u)$.

If $(v, u)$ is an up-edge, then $e_{G}^{M}(u)=e_{G}^{M}(v)+1, \mathbb{U}((v, u))=1$, and $\mathbb{D}((v, u))=0=\mathbb{U}((v, u))-1$. By the inductive hypothesis, $\mathbb{D}(P(y, u))=\mathbb{D}(P(y, v))+\mathbb{D}((v, u))=\mathbb{U}(P(y, v))+e_{G}^{M}(y)-e_{G}^{M}(v)+$ $\mathbb{U}((v, u))-1=\mathbb{U}(P(y, u))+e_{G}^{M}(y)-e_{G}^{M}(v)-1=\mathbb{U}(P(y, u))+e_{G}^{M}(y)-e_{G}^{M}(u)$.

If $(v, u)$ is a horizontal-edge, then $e_{G}^{M}(u)=e_{G}^{M}(v), \mathbb{U}((v, u))=0=\mathbb{D}((v, u))$. By the inductive hypothesis, $\mathbb{D}(P(y, u))=\mathbb{D}(P(y, v))+\mathbb{D}((v, u))=\mathbb{U}(P(y, v))+e_{G}^{M}(y)-e_{G}^{M}(v)+\mathbb{U}((v, u))=\mathbb{U}(P(y, u))+$ $e_{G}^{M}(y)-e_{G}^{M}(v)=\mathbb{U}(P(y, u))+e_{G}^{M}(y)-e_{G}^{M}(u)$.

If $(v, u)$ is a down-edge, then $e_{G}^{M}(u)=e_{G}^{M}(v)-1, \mathbb{U}((v, u))=0$, and $\mathbb{D}((v, u))=\mathbb{U}((v, u))+1$. By the inductive hypothesis, $\mathbb{D}(P(y, u))=\mathbb{D}(P(y, v))+\mathbb{D}((v, u))=\mathbb{U}(P(y, v))+e_{G}^{M}(y)-e_{G}^{M}(v)+\mathbb{U}((v, u))+1=$ $\mathbb{U}(P(y, u))+e_{G}^{M}(y)-e_{G}^{M}(v)+1=\mathbb{U}(P(y, u))+e_{G}^{M}(y)-e_{G}^{M}(u)$.

This completes the proof of (i) that $\mathbb{D}(P(y, x))-\mathbb{U}(P(y, x))=e_{G}^{M}(y)-e_{G}^{M}(x)$. To complete the proof of (ii), it now suffices to see that $\mathbb{U}(P(y, x))+\mathbb{H}(P(y, x))+\left(\mathbb{U}(P(y, x))+e_{G}^{M}(y)-e_{G}^{M}(x)\right)=\mathbb{U}(P(y, x))+$ $\mathbb{H}(P(y, x))+\mathbb{D}(P(y, x))=d_{G}(y, x)$. Hence, $2 \mathbb{U}(P(y, x))+\mathbb{H}(P(y, x))=d_{G}(y, x)-\left(e_{G}^{M}(y)-e_{G}^{M}(x)\right)$.

Theorem 8. Let $G$ be an $\alpha$-weakly-Helly graph and $M \subseteq V(G)$. For any shortest path $P(y, x)$ of $G$ from a vertex $y \notin C_{G}^{\alpha}(M)$ to a closest vertex $x \in C_{G}^{\alpha}(M), 2 \mathbb{U}(P(y, x))+\mathbb{H}(P(y, x)) \leq 2 \alpha$ holds.

Proof. Let $x \in C_{G}^{\alpha}(M)$ be closest to $y$. Hence, $e_{G}^{M}(x)=\operatorname{rad}_{G}(M)+\alpha$. Applying Theorem 5 , $e_{G}^{M}(y) \geq d_{G}\left(y, C_{G}^{\alpha}(M)\right)+\operatorname{rad}_{G}(M)-\alpha$. Hence, $d_{G}(y, x)=d_{G}\left(y, C_{G}^{\alpha}(M)\right) \leq e_{G}^{M}(y)-\operatorname{rad}_{G}(M)+\alpha=$ $e_{G}^{M}(y)-e_{G}^{M}(x)+2 \alpha$. Applying Lemma 16, we obtain the desired result.

As a consequence of Theorem 8 , at most $2 \alpha(G)$ non-descending edges can occur along every shortest path from any vertex $y \notin C^{\alpha}(G)$ to $C^{\alpha}(G)$. Hence, in any shortest path to $C^{\alpha}(G)$, the number of vertices with locality more than 1 does not exceed $2 \alpha(G)$. These kind of results were only known for chordal graphs and distance-hereditary graphs (at most one non-descending edge, i.e., horizontaledge [22, 26]) and for $\delta$-hyperbolic graphs (at most $4 \delta$ non-descending edges [23]). 


\section{Classes of graphs having small Helly-gap}

Let $G$ be a graph and let $\alpha:=\alpha(G)$ be its Helly-gap. We now focus on the upper bound on $\alpha$ for some special graph classes and identify those classes which exhibit a topological Helly-likeness, that is, classes which have small $\alpha$. Recall that, by definition, Helly graphs are 0-weakly-Helly. It was also recently shown that each Helly vertex in the injective hull of a distance-hereditary graph is adjacent to a real vertex [35]; thus distance-hereditary graphs are 1-weakly-Helly (this result follows also from an earlier result on existence of $r$-dominating cliques in distance-hereditary graphs [18]). Distance-hereditary graphs can be defined as the graphs where each induced path is a shortest path.

We consider several other graph classes and summarize our findings in Table 1 .

\begin{tabular}{|l|l|}
\hline Graph class & Helly-gap \\
\hline Helly & $\alpha(G)=0$ \\
\hline Distance-hereditary & $\alpha(G) \leq 1$ \\
\hline$k$-Chordal & $\alpha(G) \leq\lfloor k / 2\rfloor$ \\
\hline Chordal & $\alpha(G) \leq 1$ \\
\hline AT-free & $\alpha(G) \leq 2$ \\
\hline$n \times n$ Rectilinear grid & $\alpha(G)=1$ \\
\hline$\delta$-Hyperbolic & $\alpha(G) \leq 2 \delta$ \\
\hline Cycle $C_{n}$ & $\alpha(G)=\lfloor n / 4\rfloor$ \\
\hline$\alpha_{i}$-Metric & $\alpha(G) \leq\lceil i / 2\rceil$ \\
\hline Tree-breadth $t b(G)$ & $\alpha(G) \leq t b(G)$ \\
\hline Tree-length $t l(G)$ & $\alpha(G) \leq t l(G)$ \\
\hline Tree-width $t w(G)$ & no relation \\
\hline Bridged & unbounded \\
\hline
\end{tabular}

Table 1: The bound on the Helly-gap $\alpha(G)$ for various graph classes.

\subsection{Rectilinear grids}

Let $G$ be an $n \times n$ rectilinear grid (the cartesian product of two paths of length $n$ ). $G$ can be isometrically embedded into a $2 n \times 2 n$ king grid $H$ (the strong product of two paths of length $2 n$, and a natural subclass of Helly graphs) wherein the four extreme vertices of $G$ are the midpoints of each side of $H$ (see Fig. 6). One obtains $\mathcal{H}(G)$ by removing from $H$ any peripheral vertices that are not present in $G$. Therefore, each Helly vertex $h$ of $\mathcal{H}(G)$ corresponds to one of the $n^{2}$ squares of the grid $G$, where $h$ is adjacent to the four corners of the square and to each of the Helly vertices corresponding to the 2-4 adjacent squares. Hence, the Helly-gap of $G$ is 1 .

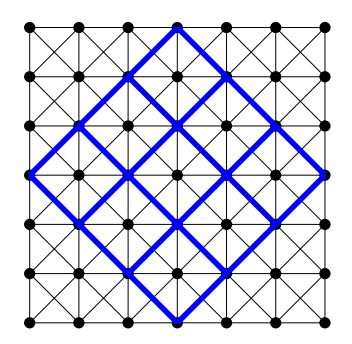

Figure 6: An $n \times n$ rectilinear grid (shown in bold blue lines) is isometrically embedded into a $2 n \times 2 n$ king grid (shown in thin black lines). 


\subsection{Graphs of bounded hyperbolicity}

Gromov [33] defines $\delta$-hyperbolic graphs via a simple 4-point condition: for any four vertices $u, v, w, x$, the two larger of the three distance sums $d(u, v)+d(w, x), d(u, w)+d(v, x)$, and $d(u, x)+d(v, w)$ differ by at most $2 \delta \geq 0$. The smallest value $\delta$ for which $G$ is $\delta$-hyperbolic is called the hyperbolicity of $G$ and is denoted by $\delta(G)$. As we have mentioned earlier, we omit $G$ from subindices when the graph is known by context.

Recall that $\kappa(G)$ denotes the smallest value $\kappa$ for which all intervals of $G$ are $\kappa$-thin. The following lemma is a folklore and easy to show using the definition of hyperbolicity.

Lemma 17. For any graph $G, \kappa(G) \leq 2 \delta(G)$.

Proof. Consider any interval $I(x, y)$ in $G$ and arbitrary two vertices $u, v \in S_{k}(x, y)$. Consider the three distance sums $S_{1}=d(x, y)+d(u, v), S_{2}=d(x, u)+d(y, v), S_{3}=d(x, v)+d(y, u)$. As $u, v \in S_{k}(x, y)$, we have $S_{2}=S_{3}=d(x, y) \leq S_{1}$. Hence, $2 \delta(G) \geq S_{1}-S_{2}=d(x, y)+d(u, v)-d(x, y)=d(u, v)$ for any two vertices from the same slice of $G$, i.e., $2 \delta(G) \geq \kappa(G)$.

We now show that the Helly-gap of any graph $G$ is at most $\kappa(\mathcal{H}(G))$.

Lemma 18. For any vertex $h \in V(\mathcal{H}(G))$ there is a real vertex $v \in V(G)$ such that $d(h, v) \leq \kappa(\mathcal{H}(G))$.

Proof. Let $H:=\mathcal{H}(G)$ and consider a vertex $h \in V(H)$. By Proposition 2, $h$ belongs to a shortest path $P_{H}(x, y)$ where $x, y \in V(G)$. Let $d_{H}(x, h)=\ell$. Because $G$ is isometric in $H$, there is a shortest path $P_{G}(x, y):=\left(v_{0}, \ldots, v_{k}\right)$, where $x=v_{0}, y=v_{k}$ and all $v_{i}$ in $P_{G}$ are real vertices. By assumption, $d_{H}\left(h, v_{\ell}\right) \leq \kappa(H)$.

Corollary 8. Any graph $G$ is $\alpha$-weakly-Helly for $\alpha \leq \kappa(\mathcal{H}(G))$.

Proof. The result follows from Theorem 1 and Lemma 18.

Note that an $n \times n$ rectilinear grid gives an example of a graph where $\alpha=1$ and $\kappa(\mathcal{H}(G))=2 n$.

Urs Lang [40] has established that the $\delta$-hyperbolicity of a graph $G$ is preserved in $H=\mathcal{H}(G)$. As hyperbolicity is preserved in $\mathcal{H}(G)$, by Lemma 18 and Lemma 17, for any Helly vertex $h \in V(H)$ there is a real vertex $v \in V(G)$ such that $d_{H}(h, v) \leq \kappa(\mathcal{H}(G)) \leq 2 \delta(\mathcal{H}(G))=2 \delta(G)$. Hence, $\delta$-hyperbolic graphs are $2 \delta$-weakly-Helly. The latter result follows also from [14].

Remark. Several graph classes are $\delta$-hyperbolic for a bounded value $\delta$, including $k$-chordal graphs and graphs without an asteroidal triple (AT). Three pairwise non-adjacent vertices of a graph form an AT if every two of them are connected by a path that avoids the neighborhood of the third. A graph is $k$-chordal provided it has no induced cycle of length greater than $k$. It is known [49] that $k$ chordal graphs have hyperbolicity at most $\frac{\lfloor k / 2\rfloor}{2}$. Therefore, $k$-chordal graphs are $\lfloor k / 2\rfloor$-weakly-Helly. Chordal graphs, which are exactly the 3-chordal graphs, are 1-weakly-Helly (this result follows also from an earlier result on existence of $r$-dominating cliques in chordal graphs [20]). The 2-weakly-Helly graphs also include all 5-chordal graphs such as AT-free graphs, all 4-chordal graphs such as weaklychordal and cocomparability graphs, as well as all 1-hyperbolic graphs including interval graphs and permutation graphs. The definitions of graph classes not given here can be found in [6].

Remark. Observe that $\alpha(G)$ can be arbitrarily smaller than the $\delta$-hyperbolicity of a graph. Consider a $(2 r \times 2 r)$ king grid $G$, i.e., the strong product of two paths each of even length $2 r$. King grids form a natural subclass of Helly graphs, and therefore $\mathcal{H}(G)=G$. Thus, $G$ is 0-weakly-Helly, whereas $\delta(G)=r$. 


\subsection{Cycles}

Recall that $C_{n}$ denotes a cycle of length $n$.

Lemma 19. The Helly-gap of a cycle $C_{n}$ is $\lfloor n / 4\rfloor$.

Proof. Let $G$ be a cycle of length $n$. Clearly, $\operatorname{diam}(G)=\lfloor n / 2\rfloor=\operatorname{rad}(G)$. By Proposition 5 , $G$ is $\alpha$ weakly-Helly for $\alpha \leq\lfloor\operatorname{diam}(G) / 2\rfloor=\lfloor n / 4\rfloor$. By Lemma 8, $\alpha \geq\lfloor(2 \operatorname{rad}(G)-\operatorname{diam}(G)) / 2\rfloor=\lfloor n / 4\rfloor$.

It should also be noted that for a self-centered graph (i.e., a graph where all vertex eccentricities are equal), from $\lfloor(2 \operatorname{rad}(G)-\operatorname{diam}(G)) / 2\rfloor \leq \alpha(G) \leq\lfloor\operatorname{diam}(G) / 2\rfloor$ and since for a self-centered graph $G, \operatorname{rad}(G)=\operatorname{diam}(G)$, we get $\alpha(G)=\lfloor\operatorname{diam}(G) / 2\rfloor=\lfloor\operatorname{rad}(G) / 2\rfloor$. Cycles are self-centered graphs.

\subsection{Graphs with an $\alpha_{i}$-metric}

Introduced by Chepoi and Yushmanov [9, 51, a graph $G$ is said to have an $\alpha_{i}$-metric if it satisfies the following: for any $x, y, z, v \in V(G)$ such that $z \in I(x, y)$ and $y \in I(z, v), d_{G}(x, v) \geq d_{G}(x, y)+$ $d_{G}(y, v)-i$ holds. For every graph $G$ with an $\alpha_{i}$-metric, $\operatorname{diam}(G) \geq 2 \operatorname{rad}(G)-i-1$ holds [51]. Several graph classes have an $\alpha_{i}$-metric [51. Ptolemaic graphs are exactly the graphs with $\alpha_{0}$-metric [51]. Chordal graphs are a subclass of graphs with $\alpha_{1}$-metric [9]. All graphs with $\alpha_{1}$-metric are characterized in [51. In a private communication, discussing the results of this paper, Victor Chepoi asked if graphs with an $\alpha_{i}$-metric are $\alpha$-weakly-Helly for an $\alpha$ depending only on $i$. The following lemma answers this question in the affirmative.

Lemma 20. Any graph $G$ with an $\alpha_{i}$-metric is $\alpha$-weakly-Helly for $\alpha \leq\lceil i / 2\rceil$.

Proof. We prove by induction on the number $k$ of disks in a family of pairwise intersecting disks $\mathcal{F}=$ $\{D(v, r(v)): v \in M \subseteq V(G)\}$. Let $y \in M$ and pick a vertex $c$ which belongs to $\bigcap_{v \in M \backslash\{y\}} D(v, r(v)+\alpha)$ such that $c$ is closest to $y$. If $d(c, y) \leq r(y)+\alpha$, then $c$ is common to all disks of $\mathcal{F}$ inflated by $\alpha$ and we are done. Assume now that $d(c, y)>r(y)+\alpha$. Let $c^{\prime} \in S_{1}(c, y)$. By choice of $c$, there is a disk $D(x, r(x)) \in \mathcal{F}$ such that $d(c, x)=r(x)+\alpha=d\left(c^{\prime}, x\right)-1$. Hence, $c \in I\left(x, c^{\prime}\right)$ and $c^{\prime} \in I(c, y)$. Applying $\alpha_{i}$-metric to $x, c, c^{\prime}, y$, one obtains $d(x, y) \geq d(x, c)+d(c, y)-i>(r(x)+\alpha)+(r(y)+\alpha)-i=$ $r(x)+r(y)+2 \alpha-i$. If now $i \leq 2 \alpha$, we get $d(x, y)>r(x)+r(y)$, contradicting the fact that disks $D(x, r(x))$ and $D(y, r(y))$ intersect. Thus, for every $i$ (even or odd) such that $i \leq 2 \alpha, d(c, y) \leq r(y)+\alpha$ must hold. That is, $G$ is $\alpha$-weakly-Helly for $\alpha=\lceil i / 2\rceil$ (pick $\alpha=i / 2$, when $i$ is even, and $\alpha=(i+1) / 2$, when $i$ is odd).

Remark. Observe that a graph $G$ with an $\alpha_{i}$-metric can have Helly-gap $\alpha(G)$ that is arbitrarily smaller than $\lceil i / 2\rceil$. Consider a $(1 \times \ell)$ rectilinear grid $G$. Let $(x, y)$ and $(z, v)$ be edges on extreme ends of $G$ so that $d(x, z)=d(y, v)=\ell$ and $d(y, z)=d(x, v)=\ell+1$. Then, $z \in I(x, v), v \in I(z, y)$, and $1=d(x, y) \geq d(x, v)+d(v, y)-i=2 \ell+1-i$. Thus, $G$ has an $\alpha_{i}$-metric for $i \geq 2 \ell$ whereas $\alpha(G)=1$.

\subsection{Graphs of bounded tree-breadth, tree-length, or tree-width}

A tree-decomposition $(\mathcal{T}, T)$ [48] for a graph $G=(V, E)$ is a family $\mathcal{T}=\left\{B_{1}, B_{2}, \ldots\right\}$ of subsets of $V$, called bags, such that $\mathcal{T}$ forms a tree $T$ with the bags in $\mathcal{T}$ as nodes which satisfy the following conditions: (i) each vertex is contained in a bag, (ii) for each edge $(u, v) \in E, \mathcal{T}$ has a bag $B$ with $u, v \in B$, and (iii) for each vertex $v \in V$, the bags containing $v$ induce a subtree of $T$. The width of a tree decomposition is the size of its largest bag minus one. A tree decomposition has breadth $\rho$ if, for each bag $B$, there is a vertex $v$ in $G$ such that $B \subseteq D_{G}(v, \rho)$. A tree decomposition has length $\lambda$ if the diameter in $G$ of each bag $B$ is at most $\lambda$. The tree-width $t w(G)$ [48, tree-breadth $t b(G)[25]$ and tree-length $\operatorname{tl}(G)$ [15] are the minimum width, breadth, and length, respectively, among all possible tree decompositions of $G$. By definition, $t b(G) \leq t l(G) \leq 2 t b(G)$, as for any graph $G$ and any set $M \subseteq V(G), \operatorname{rad}_{G}(M) \leq \operatorname{diam}_{G}(M) \leq 2 \operatorname{rad}_{G}(M)$ holds. 
Lemma 21. Any graph $G$ is $\alpha$-weakly-Helly for $\alpha \leq t b(G)$ and $\alpha \leq t l(G)$.

Proof. Let $G$ have tree-breadth $t b(G) \leq \rho$. Consider a corresponding tree-decomposition $(\mathcal{T}, T)$ of $G$ of breadth $\rho$, and let $\mathcal{F}=\left\{D_{G}(v, r(v)): v \in S \subseteq V(G)\right\}$ be a family of disks of $G$ that pairwise intersect. Observe that bags containing vertices of a disk induce a subtree of $T$ and the subtrees of $T$ corresponding to disks of $\mathcal{F}$ pairwise intersect in $T$. If the subtrees of a tree pairwise intersect, then they have a common node in $T$. Therefore, there is a bag $B \in \mathcal{T}$ such that each disk in $\mathcal{F}$ intersects $B$ in $G$. Let vertex $w$ be the center of bag $B$, i.e., $B \subseteq D_{G}(w, \rho)$. So, each $v \in S$ satisfies $w \in D_{G}(v, r(v)+\rho)$. Hence, $G$ is $\alpha$-weakly-Helly where $\alpha \leq t b(G) \leq t l(G)$.

Remark. While $\alpha:=\alpha(G)$ is upper bounded by tree-breadth and tree-length, $\alpha$ can be arbitrarily far from tree-width and arbitrarily smaller than tree-length. Consider the $(r \times r)$ rectilinear grid $G$ which is 1-weakly-Helly but $t w(G)=r$ (cf. [47]) and $t l(G)=2 r$ (cf. [1,15]). On the other hand, let $G$ be a cycle of size $4 k$; the Helly-gap of $G$ is $k$, yet $t w(G)=2$.

\subsection{Bridged graphs}

A graph is bridged [32] if it contains no isometric cycles of length greater than 3. As any isometric subgraph is an induced subgraph, bridged graphs form a superclass of chordal graphs. Interestingly, we find that although chordal graphs are 1-weakly-Helly, the Helly-gap of a bridged graph can be arbitrarily large. Consider the bridged graph $G$ in Fig. 7 with each side of length $s=4 k$ for some integer $k$. Clearly the disks centered at $x, y, z$ with radius $2 k$ pairwise intersect and have no common intersection. However, only extending all radii by $k$ yields middle vertex $u$ as a common intersection. Thus, the Helly-gap of $G$ is at least $k$, where $k$ can be arbitrarily large.

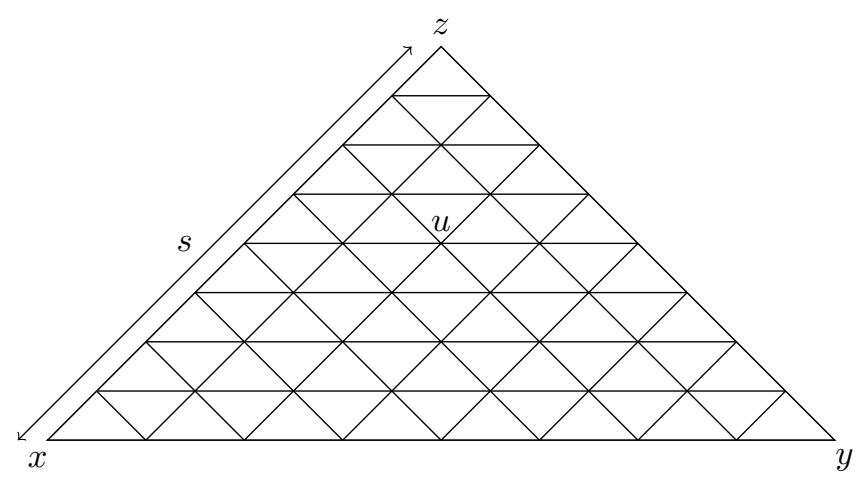

Figure 7: Example that bridged graphs have arbitrarily large Helly-gap.

\section{Conclusion}

We introduced a new metric parameter for a graph, the Helly-gap $\alpha(G)$. We characterized $\alpha$-weaklyHelly graphs with respect to distances in their injective hulls and used this as a tool to generalize many eccentricity related results known for Helly graphs to much larger family of graphs, $\alpha$-weakly-Helly graphs. In particular, we related the diameter, radius, center, and all eccentricities in $G$ to their counterparts in $\mathcal{H}(G)$. We provided estimates on $\alpha(G)$ and on eccentricities based on a variety of conditions, thereby generalizing some eccentricity related results known not only for Helly graphs but also for distance-hereditary graphs, chordal graphs, and $\delta$-hyperbolic graphs. Several additional graph classes are identified which have a bounded Helly-gap.

Two interesting questions remain open. First, is there a characterization of $\alpha(G)$ using only the radius and diameter of every $M \subseteq V(G)$ ? Is it true that $\alpha(G)=\max _{M \subseteq V(G)}\left\lfloor\left(2 \operatorname{rad}_{G}(M)-\right.\right.$ $\left.\left.\operatorname{diam}_{G}(M)\right) / 2\right\rfloor$ ? As it was recently shown [21], the equality $\max _{M \subseteq V(G)}\lfloor(2 \operatorname{rad} G(M)-$ 
$\left.\left.\operatorname{diam}_{G}(M)\right) / 2\right\rfloor=0$ characterizes the Helly graphs (i.e., the graphs with $\alpha(G)=0$ ): $G$ is a Helly graph if and only if $\max _{M \subseteq V(G)}\left\lfloor\left(2 \operatorname{rad}_{G}(M)-\operatorname{diam}_{G}(M)\right) / 2\right\rfloor=0$. Second, as $\alpha$-weakly-Helly graphs are a far reaching superclass of $\delta$-hyperbolic graphs, under what additional conditions are $\alpha$-weaklyHelly graphs reduced to $\delta$-hyperbolic graphs for some $\delta$ dependent on $\alpha$ ?

Acknowledgment: We are grateful to Victor Chepoi for reading an earlier version of this paper and providing a few interesting remarks.

\section{References}

[1] A. B. Adcock, B. D. Sullivan, and M. W. Mahoney. Tree decompositions and social graphs. Internet Mathematics, 12(5):315-361, May 2016.

[2] N. Alon, G. Kalai, J. Matouek, and R. Meshulam. Transversal numbers for hypergraphs arising in geometry. Advances in Applied Mathematics, 29(1):79 - 101, 2002.

[3] N. Alon and D. J. Kleitman. Piercing convex sets and the Hadwiger-Debrunner (p, q)-problem. Advances in Mathematics, 96(1):103 - 112, 1992.

[4] H.-J. Bandelt and E. Pesch. Dismantling absolute retracts of reflexive graphs. Eur. J. Comb., 10(3):211-220, May 1989.

[5] H.-J. Bandelt and E. Prisner. Clique graphs and helly graphs. Journal of Combinatorial Theory, Series B, 51(1):34 - 45, 1991.

[6] A. Brandstdt, V. B. Le, and J. P. Spinrad. Graph Classes: A Survey. Society for Industrial and Applied Mathematics, 1999.

[7] I. Brny and J. Matouek. A fractional Helly theorem for convex lattice sets. Advances in Mathematics, 174(2):227 - 235, 2003.

[8] J. Chalopin, V. Chepoi, A. Genevois, H. Hirai, and D. Osajda. Helly groups. arXiv:2002.06895, 2020 .

[9] V. Chepoi. Centers of triangulated graphs. Mathematical Notes of the Academy of Sciences of the USSR, 43:8286, 1988.

[10] V. Chepoi. A note on r-dominating cliques. Discret. Math., 183(1-3):47-60, 1998.

[11] V. Chepoi, F. Dragan, B. Estellon, M. Habib, and Y. Vaxès. Diameters, centers, and approximating trees of delta-hyperbolic geodesic spaces and graphs. Symposium on Computational Geometry, 2008.

[12] V. Chepoi and F. F. Dragan. A linear-time algorithm for finding a central vertex of a chordal graph. In Algorithms - ESA, pages 159-170, 1994.

[13] V. Chepoi, F. F. Dragan, M. Habib, Y. Vaxès, and H. Alrasheed. Fast approximation of eccentricities and distances in hyperbolic graphs. J. Graph Algorithms Appl., 23(2):393-433, 2019.

[14] V. Chepoi and B. Estellon. Packing and covering delta-hyperbolic spaces by balls. In $A P P R O X$ RANDOM, pages 59-73, 2007.

[15] Y. Dourisboure and C. Gavoille. Tree-decompositions with bags of small diameter. Discrete Mathematics, 307(16):2008 - 2029, 2007. EuroComb '03 - Graphs and Algorithms. 
[16] F. F. Dragan. Centers of Graphs and the Helly Property (in Russian). PhD thesis, Moldava State University, Chişinău, 1989.

[17] F. F. Dragan. Conditions for coincidence of local and global minima for eccentricity function on graphs and the Helly property (in Russian). Studies in Applied Mathematics and Information Science, pages 49-56, 1990.

[18] F. F. Dragan. Dominating cliques in distance-hereditary graphs. In Algorithm Theory - SWAT, pages 370-381, 1994.

[19] F. F. Dragan. An eccentricity 2-approximating spanning tree of a chordal graph is computable in linear time. Inf. Process. Lett., 154, 2020.

[20] F. F. Dragan and A. Brandstädt. r-dominating cliques in graphs with hypertree structure. Discret. Math., 162(1-3):93-108, 1996.

[21] F. F. Dragan, G. Ducoffe, and H. M. Guarnera. Fast deterministic algorithms for computing all eccentricities in (hyperbolic) Helly graphs. Manuscript, 2020.

[22] F. F. Dragan and H. M. Guarnera. Eccentricity function in distance-hereditary graphs. CoRR, abs/1907.05445, 2019.

[23] F. F. Dragan and H. M. Guarnera. Eccentricity terrain of $\delta$-hyperbolic graphs. Journal of Computer and System Sciences, 2020.

[24] F. F. Dragan, M. Habib, and L. Viennot. Revisiting radius, diameter, and all eccentricity computation in graphs through certificates. CoRR, abs/1803.04660, 2018.

[25] F. F. Dragan and E. Köhler. An approximation algorithm for the tree t-spanner problem on unweighted graphs via generalized chordal graphs. Algorithmica, 69(4):884-905, 2014.

[26] F. F. Dragan, E. Köhler, and H. Alrasheed. Eccentricity approximating trees. Discrete Applied Mathematics, 232:142-156, 2017.

[27] A. W. Dress. Trees, tight extensions of metric spaces, and the cohomological dimension of certain groups: A note on combinatorial properties of metric spaces. Advances in Mathematics, 53(3):321 $-402,1984$.

[28] G. Ducoffe. Facility Location problems on Helly graphs and k-Helly graphs. Manuscript, 2020.

[29] G. Ducoffe and F. F. Dragan. A story of diameter, radius and Helly property. CoRR, abs/1910.10412, 2019.

[30] J. Eckhoff. Chapter 2.1 - Helly, Radon, and Carathodory Type Theorems. In P. Gruber and J. Wills, editors, Handbook of Convex Geometry, pages 389 - 448. North-Holland, Amsterdam, 1993.

[31] R. Espínola and M. A. Khamsi. Introduction to Hyperconvex Spaces, pages 391-435. Springer Netherlands, Dordrecht, 2001.

[32] M. Farber. Bridged graphs and geodesic convexity. Discrete Mathematics, 66(3):249 - 257, 1987.

[33] M. Gromov. Hyperbolic Groups, pages 75-263. Springer, New York, NY, 1987.

[34] M. Groshaus, M. C. Lin, and J. L. Szwarcfiter. On neighborhood-Helly graphs. Discret. Appl. Math., 216:191-202, 2017. 
[35] H. M. Guarnera, F. F. Dragan, and A. Leitert. Injective hulls of various graph classes. Manuscript, 2020.

[36] J. Isbell. Six theorems about injective metric spaces. Commentarii mathematici Helvetici, 39:65$76,1964$.

[37] M. Katchalski and A. Liu. A problem of geometry in $R^{n}$. Proceedings of the American Mathematical Society, 75(2):284-288, 1979.

[38] M. Khamsi, H. Knaust, N. Nguyen, and M. O’Neill. Lambda-hyperconvexity in metric spaces. Nonlinear Analysis: Theory, Methods 83 Applications, 43(1):21 - 31, 2001.

[39] D. Kratsch, H. Le, H. Müller, E. Prisner, and D. Wagner. Additive tree spanners. SIAM Journal on Discrete Mathematics, 17(2):332-340, 2003.

[40] U. Lang. Injective hulls of certain discrete metric spaces and groups. Journal of Topology and Analysis, 05(03):297-331, 2013.

[41] W. Lenhart, R. Pollack, J. Sack, R. Seidel, M. Sharir, S. Suri, G. T. Toussaint, S. Whitesides, and C. Yap. Computing the link center of a simple polygon. Discret. Comput. Geom., 3:281-293, 1988.

[42] M. C. Lin and J. L. Szwarcfiter. Faster recognition of clique-Helly and hereditary clique-Helly graphs. Inf. Process. Lett., 103(1):40-43, 2007.

[43] R. Nandakumar and K. Parthasarathy. Eccentricity-preserving spanning trees. J. Math. Phys Sci, 24:33-36, 1990.

[44] R. Nowakowski and I. Rival. The smallest graph variety containing all paths. Discrete Mathematics, 43(2):223 - 234, 1983.

[45] E. Prisner. Eccentricity-approximating trees in chordal graphs. Discrete Mathematics, 220(13):263-269, Jun 2000.

[46] A. Quilliot. On the helly property working as a compactness criterion on graphs. Journal of Combinatorial Theory, Series A, 40(1):186 - 193, 1985.

[47] N. Robertson and P. Seymour. Graph minors. iii. planar tree-width. Journal of Combinatorial Theory, Series B, 36(1):49 - 64, 1984.

[48] N. Robertson and P. Seymour. Graph minors. ii. algorithmic aspects of tree-width. Journal of Algorithms, 7(3):309 - 322, 1986.

[49] Y. Wu and C. Zhang. Hyperbolicity and chordality of a graph. The Electronic Journal of Combinatorics, 18(1):P43, 2011.

[50] H.-G. Yeh and G. J. Chang. Centers and medians of distance-hereditary graphs. Discrete Math., 265(13):297310, Apr. 2003.

[51] S. Yushmanov and V. Chepoi. A general method of investigation of metric graph properties related to the eccentricity (in Russian). Mathematical Problems of Cybernetics (Moscow), 3:217$232,1991$. 